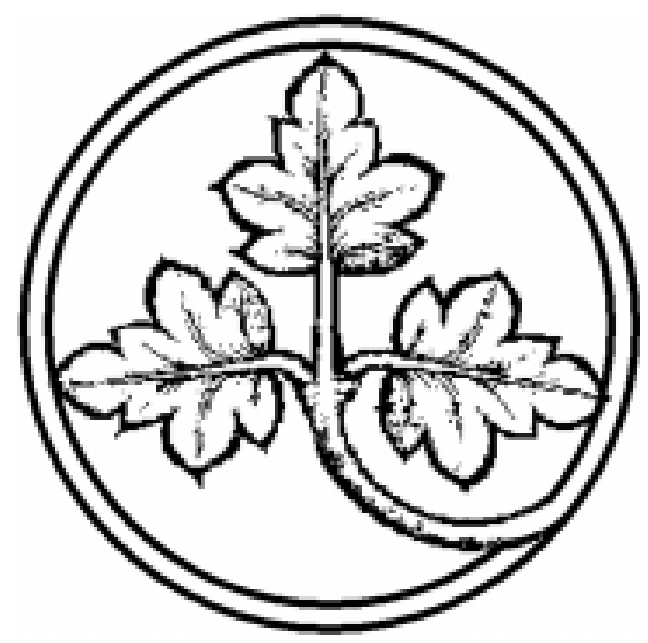

Gemeinschaftsgüter: Recht, Politik und Ökonomie

Preprints
aus der Max-Planck-Projektgruppe
Recht der Gemeinschaftsgüter
Bonn
$2001 / 15$

Die Wirkung internationaler Institutionen:

Von der Normanerkennung zur Normeinhaltung

Von

Tanja A. Börzel/Thomas Risse 


\section{Die Wirkung internationaler Institutionen: Von der Normanerkennung zur Normeinhaltung \\ von}

Tanja A. Börzel und Thomas Risse 


\section{Einleitung}

Die empirische und theoretische Forschung zur Rolle internationaler Institutionen in der Weltpolitik hat sich in der Vergangenheit vor allem auf die Entstehung internationaler Regelsysteme konzentriert und dabei die Wirkungen dieser Normen und Regeln auf das Verhalten der Akteure vernachlässigt. Implizit war dies die Folge der Dominanz rationalistischer Ansätze: Warum sollten Staaten erhebliche Anstrengungen zur Aushandlung von internationalen Institutionen unternehmen, wenn sie sich anschließend nicht an die Regeln halten? Erst in den letzten Jahren hat sich ein Forschungszweig herausentwickelt, der sich weniger mit der Entstehung und Weiterentwicklung internationaler Institutionen beschäftigt, sondern deren Wirkung auf staatliches Verhalten analysiert. Unser Beitrag knüpft an diese Arbeiten an. Wir konzentrieren uns auf Mechanismen der Institutionenwirkung, die wir als Prozess begreifen, der von der Normanerkennung zur Normeinhaltung führt. Nach einer Begriffsklärung der Normeinhaltung als ,,regelkonformes Verhalten“ (compliance) unterscheiden wir zwischen zwei sozialen Logiken der Institutionenwirkung. Beide Perspektiven gehen davon aus, dass nur „unbequeme Regeln“ Probleme bei der Normeinhaltung verursachen, weil sie materielle oder ideelle Kosten verursachen bzw. an gegebene Institutionen oder Identitäten nicht anschlussfähig sind. Rationalistische Ansätze konzentrieren sich dabei auf die Verhaltenssteuerung durch positive und negative Anreize. Wir unterscheiden vier rationalistische Erklärungsfaktoren der Normeinhaltung. Erstens stellen internationale Institutionen Sanktionierungsmechanismen bereit, welche die Kosten von Regelbrüchen wirksam erhöhen können (Legalisierungshypothese). Zweitens stärken Ressourcentransfers die administrativen und politischen Handlungskapazitäten betroffener Staaten und erleichtern so die Einhaltung unbequemer Regeln (Management-Hypothese). Drittens ist die Autonomie nationaler Regierungen gegenüber ihren gesellschaftlichen Umfeldern entscheidend für die Fähigkeit, unbequeme Regeln innerstaatlich durchzusetzen (liberal-institutionenzentrierte Hypothese). Schließlich wird die Normeinhaltung dadurch befördert, dass institutionelle Regeln die innenpolitischen Kräfteverhältnisse zugunsten derjenigen Akteure verändern, die von den internationalen Normen profitieren (liberal-akteurszentrierte Hypothese). Demgegenüber beschreiben Ansätze des konstruktivistischen oder soziologischen Institutionalismus die Normeinhaltung als einen Prozess, bei dem die Internalisierung von Normen zu Präferenzänderungen der Akteure führt. Auch hier formulieren wir vier Hypothesen zur Normeinhaltung. Erstens erhöht die Einbeziehung der Regelzielgruppen in das Verfahren der Normfindung die Legitimität internationaler Regeln und damit die Folgebereitschaft (Verfahrenslegitimitäts-Hypothese). Einen ähnlichen Effekt bewirkt, zweitens, die Einbettung einer Regel in umfassendere und weithin als legitim angesehene Institutionen (institutionelle Legitimitäts-Hypothese). Drittens ermöglicht die unmittelbare Rechtswirkung und die Einklagbarkeit einer Regel einen rechtlichen Diskurs über ihre Bedeutung und ihre Geltung (rechtliche Internalisierungs-Hypothese). Schließlich stoßen transnationale oder nationale „Normunternehmer“ gesellschaftliche Lern- und Überzeugungsprozesse an, durch die internationale Regeln von ihren Adressaten und Zielgruppen neue Regeln als „selbstverständlich“ in ihr Handlungsrepertoire übernehmen (soziale Internalisierungs-Hypothese). 
Obwohl rationalistische und konstruktivistische Ansätze zur Institutionenwirkung sich grundsätzlich im Hinblick auf die jeweils unterstellten sozialen Handlungslogiken unterscheiden, schließen sich die daraus ableitbaren Hypothesen zur Normeinhaltung keinesfalls gegenseitig aus, sondern lassen sich auf vielfältige Weise zueinander in Beziehung setzen. Wir illustrieren dies anhand empirischer Beispiele aus dem Bereich der europäischen Umweltpolitik einerseits und der Einhaltung internationaler Menschenrechte andererseits. Unser Beitrag schließt mit einigen Vorschlägen für die künftige Forschung zur Frage der Institutionenwirkungen.

\section{Von der Normanerkennung zur Normeinhaltung: Logiken der Institutionenwirkung}

\subsection{Was ist regelkonformes Verhalten (compliance)?}

Die Anerkennung einer internationalen Norm oder Regel erfolgt, wenn sie Geltung im nationalen Recht erlangt. Im Völkerrecht bedeutet dies gewöhnlich die Ratifizierung eines internationalen Abkommens durch ein nationales Gesetz. Im Europarecht erfolgt die Normanerkennung unmittelbar mit der Normbildung, da europäische Regeln und Normen Vorrang vor dem nationalen Recht genießen und meistens direkt anwendbar sind, auch wenn sie der Umsetzung ins nationale Recht bedürfen. Die Normanerkennung bedingt jedoch keinesfalls zwingend die Normeinhaltung (compliance). Nur weil eine internationale oder europäische Regel rechtliche Geltung hat, wird sie faktisch noch keinesfalls angewendet und befolgt.

In der einschlägigen Literatur wird Normeinhaltung gewöhnlich als regelkonformes Verhalten definiert (Young 1979; Victor, et al. 1998; Mitchell 1994a). Die meisten compliance-Studien konzentrieren sich auf das regelkonforme Verhalten von Staaten bzw. deren Regierungen und vernachlässigen dabei die Rolle von nicht-staatlichen Akteuren. Um der Bedeutung privater Akteure gerecht $\mathrm{zu}$ werden, ist es sinnvoll, zwischen regelkonformem Verhalten der Adressaten (rule addressees) und dem der Zielgruppe (rule targets) einer Regel zu unterscheiden. Während sich die Regeln des Völker- und Gemeinschaftsrechts gewöhnlich an Staaten richten, sind diese nicht notwendigerweise ihre Hauptzielgruppe. So verstanden bezieht sich compliance dann auf das regelkonforme Verhalten der Akteure, welche die formalen Adressaten der Regel sind und deren Verhalten durch die Regel bestimmt werden soll. Die Unterscheidung zwischen Adressaten und Zielgruppen erleichtert auch die Abgrenzung zwischen compliance einerseits und den verschieden Konzepten der Implementationsforschung andererseits. Implementation bezieht sich auf die Umsetzung einer Regel in die Praxis. In Anlehnung an den systemtheoretischen Ansatz von David Easton differenzieren Implementationsstudien häufig zwischen drei Phasen des Umsetzungsprozesses:

output: die gesetzlichen und administrativen Maßnahmen, welche die praktische Anwendung der Regel ermöglichen; 
outcome: die Wirkung der gesetzlichen und administrativen Maßnahmen auf das Verhalten der Zielgruppe;

impact: die Wirkung einer Regel auf ihr sozio-ökonomisches Umfeld (Problemlösungsfähigkeit, Effektivität).

Der hier zugrunde gelegte Begriff der Normeinhaltung umfasst die output- und die outcome- Dimensionen. Die Frage der Effektivität (impact) oder Problemlösungsfähigkeit einer Regel wird beiseite gelassen, weil regelkonformes Verhalten nicht notwendigerweise zu einer Veränderung im sozio-ökonomischen Umfeld führen muss.

Hinsichtlich des outputs erfordert regelkonformes Verhalten von den Adressaten, dass sie:

- die Regel vollständig und korrekt in nationales Recht umsetzen und entgegenstehende nationale Bestimmungen ändern oder aussetzen,

- den Verwaltungsapparat und die Ressourcen bereitstellen, die für die praktische Anwendung der Regel sowie für die Überwachung des regelkonformen Verhaltens der Zielgruppe notwendig sind,

- das regelkonforme Verhalten der Zielgruppe durch effektives Überwachen, die Bereitstellung positiver und/oder negativer Sanktionen sowie ggf. der Durchsetzung von Zwangsmaßnahmen befördern.

Hinsichtlich des outcomes erfordert regelkonformes Verhalten von der Zielgruppe, dass sie:

- Handlungen unternimmt, um ihr Verhalten den Anforderungen der Regel anzugleichen bzw.

- Handlungen unterlässt, die gegen die Regel verstoßen.

Unabhängig davon, welcher Theorie sie im einzelnen verpflichtet sind, teilen die meisten Ansätze zur Untersuchung von Institutionenwirkungen eine Grundannahme: Nur „unbequeme“ Regeln führen überhaupt zu „,compliance“-Problemen, weil deren Einhaltung Anpassungskosten verursacht, die von Regeladressanten und Regelzielgruppen selten widerstandslos hingenommen werden. Ähnlich argumentiert aus konstruktivistischer Sicht die „Resonanzhypothese“, dass neue internationale Normen umso eher innenpolitisch umgesetzt und schließlich eingehalten werden, je stärker sie an bereits existierende innenpolitische Regelsystemen, kollektive Bedeutungsgehalte oder Identitäten anschlußfähig sind (Checkel 1997; Ulbert 1997). Für die Forschung wird es daher erst interessant, wenn die Normen und Regeln internationaler Institutionen mit nationalen Standards, politischen und gesellschaftlichen Institutionen, Problemlösungsphilosophien und kollektiven Identitäten mindestens teilweise unvereinbar sind (Breitmeier und Wolf 1995, 347348; Underdal 1998, 12; Cortell und Davis 2000; Checkel 1997). Die diversen complianceTheorien unterscheiden sich dahingehend, wie sie dieses Anpassungsproblem konzeptualisieren und welche Faktoren und Mechanismen sie betonen, um regelkonformes Verhalten trotz unbequemer Normen zu erklären. 


\subsection{Rationalistischer Institutionalismus: Verhaltenssteuerung durch Anreize}

Der rationalistische (oder neoliberale) Institutionalismus folgt einer Logik der instrumentellen Rationalität. Zielorientierte Akteure versuchen, in strategischen Interaktionen ihren Nutzen zu maximieren oder zu optimieren auf der Basis von gegebenen und transitiv geordneten Präferenzen. Sie verhalten sich instrumentell rational, indem sie Kosten und Nutzen verschiedener Verhaltensweisen kalkulieren und dabei das antizipierte Verhalten ihrer Interaktionspartner in diese Kalküle einbeziehen (strategische Rationalität). Institutionen wirken auf das Verhalten instrumentell rationaler Akteure, indem sie die zur Verfügung stehenden Handlungsoptionen einschränken, wohingegen die zugrundeliegenden Interessen und Handlungsziele unberührt bleiben. Daraus folgt logisch, dass der rationalistische Institutionalismus vor allem die Verhaltenssteuerung durch positive und negative Anreize betont, um die Einhaltung unbequemer Regeln erklären zu können. Sie wird dadurch erreicht, dass die Kosten der Regelbefolgung durch positive Anreize gesenkt bzw. die Kosten von Regelverletzungen durch (die Drohung mit) Sanktionen erhöht werden.

Allerdings sehen sich rationalistische Ansätze einem theoretischen Puzzle gegenüber, wenn sie die Einhaltung unbequemer Regeln erklären wollen. Unter der Annahme vollständiger Information sollten strategisch-rationale Akteure (z.B. nationale Regierungen) die Folgen ihrer Handlungen auch in der Zukunft abschätzen können. Zumindest materiell und ideell mächtige Akteure dürften sich also gar nicht erst auf ,unbequeme” Regeln einlassen, die Kosten bei der Implementation und Einhaltung bereiten könnten. Vertreter neorealistischer Ansätze haben konsequenterweise behauptet, dass (mächtige) Staaten sich an internationale Regeln halten, weil sie ohnehin nur solche Normen vereinbaren, die sie problemlos einhalten können (Downs, et al. 1996). Dabei haben hegemoniale Staaten die Möglichkeit, unbequeme Regeln durchzusetzen, indem sie schwächere Staaten durch Sanktionsdrohungen zur Regeleinhaltung bringen und ggf. auch zwingen.

Demgegenüber betont der rationalistische Institutionalismus, dass internationale Institutionen das Kosten-Nutzen Verhältnis zwischen Regeleinhaltung und Regelverstoß verändern können (Keohane 1984; Zürn 1997; Gehring 1994). Staaten haben einerseits ein prinzipielles Interesse an der Einhaltung von Regeln, sonst hätten sie ihnen nicht zugestimmt. Andererseits haben sie einen Anreiz zur Nichtbefolgung, solange sich die anderen Staaten weiterhin an die Regeln halten. Ein solches Trittbrettfahrertum wird dann unattraktiv, wenn die Wahrscheinlichkeit des Entdeckt- und Bestraftwerdens relativ hoch ist. Die in viele internationale Regime eingebauten Überwachungs- und Sanktionsmechanismen haben die Funktion, Trittbrettfahrertum zu begrenzen, d. h. zu verhindern, dass bestimmte Akteure (vor allem kleinere Staaten) von den Regeln profitieren, ohne die Kosten der Einhaltung zu tragen. Darüber hinaus stellen internationale Institutionen Schlichtungsverfahren zur Beilegung von Konflikten über die Bedingungen der Regeleinhaltung bereit und tragen zu einer fairen Verteilung von Kosten und Nutzen der Regelbefolgung bei (vgl. Mills und Rockoff 1987; Mitchell 1996; Victor, et al. 1998). 
Wenn man die Annahmen des rationalistischen Institutionalismus und die Bedingung vollständiger Information zugrundelegt, dann ergeben sich Probleme bei der Regeleinhaltung internationaler Institutionen also vor allem dann, wenn die Regeln selbst unvollständig sind (,incomplete contracting"). Internationale Normen und Regeln sind oft nicht spezifisch genug und die Überwachungs- und Sanktionierungsmechanismen internationaler Regime sind häufig schwach ausgebildet (Abbott und Snidal 2000; Raustiala und Slaughter 2002). Dies kommt vor allem dadurch zustande, dass nationale Regierungen ihr Interesse an klaren Regeln und strengen Sanktionsmechanismen gegenüber den Kosten des damit verbundenen Verlusts an nationaler Autonomie und Souveränität ausbalancieren müssen. Schließlich sind in vielen Fällen der Regimebildung die distributiven Konflikte derart ausgeprägt, dass eine Einigung schwierig ist, Staaten unklare Regeln aber gegenüber einem völlig ungeregelten Zustand vorziehen. Die Literatur zur Verrechtlichung internationaler Normen kommt daher zu dem Schluss, daß die Einhaltung auch unbequemer Regeln umso eher zu erwarten ist, je spezifischer und präziser die Regeln und je ausgeprägter die Überwachungs- und Sanktionsmechanismen sind (Abbott, et al. 2000; Legro 1996). Die empirische Prüfung dieser Hypothese führte bisher zu keinem eindeutigen Befund. Während Studien zu diversen Umweltregimen sowie zur Einhaltung von EU-Recht die Hypothese zu bestätigen scheinen (Mitchell 1994a; Victor, et al. 1998; Alter 2000), konnten Untersuchungen zur Einhaltung von Menschenrechtsnormen in Lateinamerika den Zusammenhang nicht erhärten (Lutz und Sikkink 2000).

Im Unterschied zu denjenigen Ansätzen, die effektive Überwachungs- und Sanktionierungsmechanismen betonen, unterstellen Management-Ansätze begrenzte Rationalität (Simon 1982, 1997; Odell i.E.), also z.B. Ungewissheit über die Verhaltensanforderungen der Regel oder die vorhandenen Handlungskapazitäten. Es wird davon ausgegangen, dass Staaten grundsätzlich bereit sind, sich an internationale Regeln zu halten, selbst wenn sie Kosten verursachen. Die meisten Regelverletzungen geschehen unbeabsichtigt, weil den Staaten z. B. die notwendigen Handlungskapazitäten zur Um- und Durchsetzung oder die notwendigen Informationen über die Verhaltensanforderungen der Regel fehlen (vgl. Chayes, et al. 1998, Chayes und Chayes Handler 1993, 1995; Levy, et al. 1993; Marauhn 1996; Zürn 1997). Internationale Institutionen spielen bei der Verhütung solcher ,ungewollten Regelverstöße” eine wichtige Rolle. Ihre vorrangige Funktion liegt in der Bereitstellung finanzieller und technischer Unterstützung für die Staaten, denen es an den für die Regelumsetzung notwendigen Geldmitteln, Expertise und Technologie fehlt. Insbesondere Arbeiten über Entwicklungsländer haben die zentrale Bedeutung eines solchen „capacity building“ für die erfolgreiche Umsetzung internationaler Abkommen z.B. im Umweltbereich aufgezeigt (Ponce-Nava 1995; Beiträge in Jänicke und Weidner 1997). Neben dem finanziellen und technischen Ressourcentransfer stellen Institutionen Verfahren und Informationen zur Klärung von Regelinhalten und etwaigen Regelbrüchen bereit. Empirische Studien betonen hier die Bedeutung eines kontinuierlichen Informationsflusses über Problemlösung und Regeleinhaltung (Mitchell 1994b). Während für die einen dabei die Prozeduralisierung des Informations- und Ressourcentransfers im Vordergrund steht (Marauhn 1996), unterstreichen andere die Rolle von Expertengemeinschaften (epistemic communities) und Ideen sowie Lernprozessen bei der Entwicklung von Problemlösungen (Haas 1998; Underdal 1998). 
Die bisher diskutierten Ansätze aus der Perspektive des rationalistischen (neoliberalen) Institutionalismus folgen einer eher staatszentrierten Perspektive und behandeln Staaten als einheitliche Akteure. Ungewollte Regelverstöße können aber auch dadurch zustande kommen, dass nationale Regierungen die innenpolitischen Konsequenzen der von ihnen favorisierten internationalen Regelungen nicht absehen. Zwei Varianten dieses Arguments können dabei unterschieden werden. Die eine argumentiert institutionenorientiert und betont z. B. die Anzahl der Vetospieler in einem politischen System (Tsebelis 1995) oder die Autonomie der nationalen Regierungen gegenüber ihren gesellschaftlichen Umfeldern (Katzenstein 1984, 1978). Daraus lässt sich dann die allgemeine Hypothese ableiten, dass ungewollte Regelverstöße umso unwahrscheinlicher werden, je autonomer und handlungsfähiger nationale Regierungen gegenüber ihren gesellschaftlichen Umfeldern sind (Simmons und Martin 2002, ms., 29; Jacobson und Brown Weiss 1995).

Eine mehr akteurszentrierte Variante des Arguments rekurriert u.a. auf die Literatur zu „ZweiEbenen-Spielen“ (Putnam 1988; Evans, et al. 1993) und betont, dass innenpolitische - sowohl private wie öffentliche - Akteure häufig gegen internationale Regeln mobilisieren oder gar deren Ratifizierung verhindern (wie etwa im Falle des Teststopvertrages der amerikanische Senat). Milner und Rogowski haben umgekehrt vor mehr als zehn Jahren darauf hingewiesen, daß zunehmende ökonomische Interdependenz und die Globalisierung nationaler Märkte zur einer Veränderung der gesellschaftlichen Kräfteverhältnisse zugunsten exportorientierter Sektoren und Interessengruppen führt, die für internationale Freihandelsregime und gegen Protektionismus eintreten (Milner 1988; Rogowski 1989). Im Umweltbereich haben empirische Arbeiten gezeigt, dass die Unterstützung von gesellschaftlichen Interessengruppen von zentraler Bedeutung für die Regelbefolgung ist (Raustiala 1997; Haverland 1999; Börzel 2000). Auch in bezug auf das europäische Recht wird häufig damit argumentiert, daß die europäische Integration diejenigen politischen und gesellschaftlichen Akteure innenpolitisch stärkt, die von diesen Regelungen profitieren (Burley und Mattli 1993; Stone Sweet und Brunell 1998; Cowles, et al. 2001). Moravcsik behauptete in bezug auf die EU, dass die europäische Integration tendenziell zur Stärkung nationaler Regierungen gegenüber ihren gesellschaftlichen Umfeldern führt, und zwar über deren Kontrolle wichtiger materieller und ideeller Ressourcen (Moravcsik 1997; siehe aber dagegen Sandholtz 1996; Schmidt 1996). Ähnlich findet Goldstein, dass Regierungen mit Hilfe von internationalen Abkommen innerstaatliche Interessengruppen disziplinieren können, indem sie etwa internationale Handelabkommen zur Durchsetzung eigener Deregulierungsvorstellungen nutzen (Goldstein 1996).

Trotz ihrer zum Teil gegensätzlichen Einsichten gehen akteurszentrierte Ansätze generell davon aus, dass internationale Institutionen zu einer Umverteilung der materiellen und ideellen Ressourcen von innenpolitischen und gesellschaftlichen Akteuren führen und damit eine Veränderung der politischen und sozialen Kräfteverhältnisse bedingen können. Je mehr innenpolitische Akteure durch internationale Normen und Regeln gestärkt werden und je mehr dies zu einer Veränderung der innergesellschaftlichen Kräfteverhältnisse zugunsten einer Gewinnkoalition derer führt, die von der Regel profitieren, desto eher ist die Einhaltung auch unbequemer internationaler Regeln zu erwarten. 
Die Aussagen des rationalistischen Insitutionalismus zum Problem der Regelbefolgung lassen sich in vier Annahmen zusammenfassen. Die Einhaltung unbequemer internationaler Regeln hängt $\mathrm{ab}$ von

1. dem Ausmaß ihrer „Legalisierung“ im Sinne ihrer Spezifizität und der Dichte der Überwachungs- und Sanktionsmechanismen (Legalisierungshypothese);

2. der Stärkung administrativer und politischer Handlungskapazitäten der betroffenen Staaten (Management-Hypothese);

3. der Autonomie und Handlungsfähigkeit der politischen Systeme gegenüber ihren gesellschaftlichen Umfeldern (liberal-institutionenzentrierte Hypothese);

4. der Veränderung innenpolitischer Kräfteverhältnisse zugunsten derjenigen, die von den Regeln profitieren (liberal-akteurszentrierte Hypothese).

\subsection{Konstruktivistischer Institutionalismus: Präferenzänderung durch Normin- ternalisierung}

Auf der metatheoretischen Ebene unterscheiden sich konstruktivistische Ansätze recht grundsätzlich von rationalistischen Perspektiven (vgl. z. B. Wendt 1999; Onuf 1989; Risse i.E.). Gegen den methodologischen Individualismus von Rational Choice betonen sie, dass sich (soziale) Strukturen und Akteure wechselseitig konstituieren. Daraus folgt für unsere Fragestellung, dass soziale Normen und Regeln nicht nur verhaltenssteuernd und regulativ wirken, sondern Akteure in vielen Fällen bereits konstituieren, d.h. deren Interessen Präferenzen und sogar kollektive Identitäten verändern können. Souveränität als internationale Norm reguliert nicht nur das Verhalten von Staaten in den internationalen Beziehungen (z.B. über die Nichteinmischung in die inneren Angelegenheiten); Souveränität definiert und konstitutiert Staaten in der Weltgesellschaft im Unterschied zu anderen Akteuren (etwa multinationalen Konzernen). Darauf hat bereits die Englische Schule hingewiesen (Bull 1977). Internationale Menschenrechtsnormen definieren zunehmend, was es heisst, Mitglied der internationalen (zivilisierten) Gemeinschaft zu sein. „Zivilisierte“ Mitglieder der internationalen Gemeinschaft unterscheiden sich von „Schurkenstaaten“" u.a. durch die Einhaltung grundlegender Menschenrechte sowie den Verzicht auf die Proliferation von Massenvernichtungsmitteln oder auf die Unterstützung des internationalen Terrorismus.

Daraus folgt, dass konstruktivistische Ansätze andere soziale Handlungslogiken betonen als Vertreter der rationalistischen Perspektive. Zum einen geht es um die von March und Olsen betonte „Logik der Angemessenheit“ (logic of appropriateness, vgl. March und Olsen 1989, 1998). Normgeleitetes Verhalten unterscheidet sich von instrumentell-rationalem Verhalten dadurch, dass Akteure in einer gegebenen Situation danach streben, das sozial Angemessene und Richtige zu tun anstatt strategische Ziele zu optimieren. Sie versuchen, die für die gegebene soziale Situation angemessene Regel zu finden und sich danach zu verhalten. Wenn die Normen bekannt 
und eindeutig sind, dann werden sie gewohnheitsmäßig und quasi „,selbstverständlich“ eingehalten, ohne dass die Regeleinhaltung in jedem Fall einen bewussten Vorgang darstellt.

Zum anderen geht es um die „Logik kommunikativer Rationalität,“ auf die vor allem die in der Zeitschrift für Internationale Beziehungen über mehrere Jahre geführte Debatte hingewiesen hat (vgl. u.a. Müller 1994; Keck 1995; Zangl und Zürn 1996; Risse 2000). Häufig sind sich instrumentell-rationale Akteure über ihre eigenen Interessen im unklaren oder verfügen nicht über ausreichendes Wissen, um ihre Ziele verfolgen zu können. Ebensowenig sind sich normengeleitete Akteure in allen Fällen darüber im klaren, welche Regel die jeweils angemessene ist oder gar, ob die jeweilige Norm überhaupt moralisch vertretbar ist. In solchen Situationen bietet verständigungsorientiertes Handeln einen Ausweg. In theoretischen oder praktischen Diskursen können Akteure sich über die Situationsdefinition ebenso wie über die „richtigen“ Normen und Regeln verständigen. Argumentatives Handeln (Habermas 1981, 1992) impliziert, dass Akteure ihre Interessen und Handlungsziele hintan- oder gar zur Disposition stellen und sich gegenseitig als gleichberechtigt im Kommunikationsprozess anerkennen. Es geht also um die Durchsetzungskraft des „,besseren Arguments,“ nicht um die Verwirklichung der eigenen Präferenzen.

Konstruktivistisch oder soziologisch orientierte Institutionalisten konzeptualisieren daher das Problem der Regeleinhaltung anders als ihre rationalistischen Kollegen. Erstens wird die Anerkennung einer internationalen Norm als legitim und ihre (zumindest kognitive, wenn auch nicht unbedingt moralische) Internalisierung ${ }^{1}$ durch die Akteure zur Voraussetzung regelkonformen Verhaltens. In Lern- und Sozialisationsprozessen internalisieren Staaten und ihre Gesellschaften neue Regeln und übernehmen sie als „selbstverständlich“ in ihr Handlungsrepertoire. Dies bedeutet meist auch eine Redefinition staatlicher Präferenzen und Identitäten. Während einige Arbeiten die Rolle von internationalen Organisationen als „Lehrer“ betonen, die staatlichen Akteure bestimmte Normen und Regeln vermitteln (Finnemore 1996; Checkel 1999; vgl. auch KohlerKoch 1998a), heben andere auf die Rolle (trans)nationaler Nicht-Regierungsorganisationen ab, die staatliche Akteure mittels Argumentations- und Überzeugungsprozesse in neue Regeln und Normen hineinsozialisieren (Risse, et al. 1999).

Daraus ergibt sich, dass sich ,gewollte“ Regelverstöße nicht nur durch Zwang oder negative bzw. positive Sanktionen vermeiden lassen. Wenn staatliche Akteure von der Angemessenheit internationaler Regeln überzeugt werden und diese als Standard für international akzeptiertes Verhalten annehmen, erübrigen sich solche Maßnahmen. Statt Disziplinierung wird Überzeugung zum dominanten Mechanismus der Regeleinhaltung. Die Einbeziehung konstruktivistischer Annahmen in die Erklärung regelkonformen Verhaltens öffnet erstens den Blick für die Bedeutung der Legitimität internationaler Regeln, welche Juristen häufig betonen, die von vielen Politikwissenschaftlern aber oft vernachlässigt wird. Die Legitimität einer Regel erhöht ihre Akzeptanz und generiert damit freiwillige Folgebereitschaft (Franck 1990; Koh 1997; Joerges und Neyer 1997; Hurd 1999). Legitimität kann u.a. dadurch erzeugt werden, dass alle von der Regel

1 Norminternalisierung verlangt nicht unbedingt, dass die Regel für moralisch richtig gehalten wird, sondern lediglich, dass Akteure wissen, was von ihnen in einer gegebenen Situation als angemessenes Verhalten erwartet wird. Wir verdanken diesen Hinweis Ron Jepperson. 
potentiell betroffenen Akteure die Chance erhalten, an der Regelbildung gleichberechtigt teilzuhaben, und die Kosten und Nutzen der Regel als gerecht verteilt empfunden werden (Dworkin 1986; Franck 1995). Neben Verfahrens- und Verteilungsgerechtigkeit erhöht auch die Einbindung einer Regel in ein übergeordnetes Rechtssystem ihre Legitimität (Hurrell 1993). Ausserdem wirkt Regelbefolgung ,ansteckend“: Je mehr Staaten eine internationale Norm für legitim und angemessen halten, desto stärker wird der Druck auf diejenigen, die die Regel nach wie vor verletzen (Finnemore und Sikkink 1998; Franck 1990).

Zweitens - und ebenfalls im Einklang mit juristischen Argumentationen (vgl. z. B. Chayes und Chayes Handler 1995; Snyder 1993) - wird Regeleinhaltung nicht einfach als objektiv messbare Übereinstimmung zwischen Verhaltensanforderung und tatsächlichem Verhalten verstanden, sondern als Interpretations- und Aushandlungsprozess (vgl. auch Kratochwil 1989). Die Bedeutung und Anwendbarkeit von Regeln ergibt sich aus Auslegungsprozessen, in denen häufig zwischen konfligierenden Auffassungen vermittelt werden muss. Die Legitimität einer Norm hängt auch von einem von den Betroffenen geteilten Verständnis über ihren Bedeutungsgehalt ab. Dabei geht es nicht nur um die Präzisierung zweideutig formulierter Regeln. Häufig prallen unterschiedliche Auslegungen einer Regel und ihrer Anwendbarkeit aufeinander, zwischen denen es in einem rechtlichen Diskurs zu vermitteln gilt (Koh 1997; Marauhn 1996). Solche Vermittlungsprozesse, die häufig vor internationalen und nationalen (Schieds-) Gerichten ausgetragen werden, befördern die rechtliche Internalisierung von Regeln, die zu einer Redefinition staatlicher Interessen und Identitäten führen kann. Auch hier kommt es auf kommunikative Prozesse und die Überzeugungskraft der besseren Argumente an. Zwar argumentieren Ankläger und Verteidiger vor Gericht strategisch im Sinne rhetorischen Handelns (Schimmelfennig 1997, 2001). Nichtsdestotrotz unterliegt ihre Argumentation den Regeln verständigungsorientierten Handelns, weil sie nämlich ihre Zuhörerschaft - in diesem Fall Richter oder Jury - von ihrer Glaubwürdigkeit und der Richtigkeit der vorgetragenen Begründungen überzeugen müssen. Daraus ergibt sich, dass die Einhaltung unbequemer internationaler Regeln umso wahrscheinlicher wird, je stärker deren Rechtsqualität im nationalen Rechtssystem ausfällt und je mehr die Regel vor innerstaatlichen Gerichten einklagbar wird (rechtliche Internalisierung; vgl. Slaughter 1995; Zürn und Joerges 1999). Rechtliche Internalisierung geht daher über die bloße Umsetzung der Regel ins nationale Recht hinaus. Sie bezieht sich auf den Rechtsdiskurs über die Bedeutung und die Geltung einer international Norm im nationalen Rechtssystem.

Allerdings verweisen soziologische Institutionalisten der Stanford-Schule um John Meyer darauf, dass zwischen der Normanerkennung als legitim und der Regeleinhaltung im praktischen Verhalten eine starke Diskrepanz bestehen kann. Man spricht in diesem Zusammenhang von „Entkopplung“, die Organisationen ermöglicht, standardisierte und legitimierte formale Strukturen aufrechtzuerhalten und gleichzeitig ihre Aktivitäten aufgrund pragmatischer Erwägungen zu orientieren und zu variieren (Meyer und Rowen 1991, 58, Meyer, et al. 1987). Eine quantitative Studie fand beispielsweise keinen statistisch signifikanten Zusammenhang zwischen der Ratifizierung internationaler Menschenrechtsabkommen durch einzelne Staaten und der praktischen Einhaltung der Menschenrechte (Keith 1999). Offenbar gehört es in den 90er Jahren zum guten 
Ton, bestimmte internationale Abkommen zu unterzeichnen, wenn man als ,,anständiges Mitglied“ der internationalen Gemeinschaft gelten will. Ob man dann aber auch die entsprechenden Normen einhält, steht auf einem anderen Blatt (vgl. dazu auch Liese 2001). Das empirische Problem der „Entkopplungs“-These ist, dass sie die große Varianz bei der Regeleinhaltung nicht erklären kann. Wie kommt es, dass einige Staaten sich peinlich genau an internationale Menschenrechtsabkommen oder das EU-Recht halten, während andere eher locker damit umgehen? In der EU beispielsweise ist die Varianz zwischen den - anderweitig durchaus europaskeptischen - „Musterknaben“ Dänemark und Großbritannien einerseits und den „Bummlern“ Italien und Griechenland enorm (Börzel 2001).

Aus konstruktivistischer Perspektive wurden zwei Hypothesen entwickelt, um die Varianz bei der Regelbefolgung im praktischen Verhalten trotz verbaler und rechtlicher Normanerkennung zu erklären. Die „Resonanzhypothese“ führt die Nichteinhaltung internationaler Normen auf deren mangelnde Anschlussfähigkeit an kollektive Bedeutungsgehalte und Identitäten auf nationaler Ebene zurück. Je größer die Diskrepanz zwischen innenpolitisch institutionalisierten Normen und Regeln, desto schwieriger wird die Regeleinhaltung. Empirisch wurde diese Hypothese erfolgreich im Bereich der internationalen Umwelt- und Handelspolitik sowie des Staatsbürgerschaftsrechts evaluiert (Überblick bei Cortell und Davis 2000). Aber die Resonanzhypothese ist selbst nicht unproblematisch: Je größer die Kompatibilität zwischen internationaler Norm und innenpolitischen Regeln, umso bequemer wird die Regeleinhaltung für die Akteure. Damit hört Regelbefolgung auf, ein erklärungsbedürftiges Phänomen zu sein. Es ist daher sinnvoller, fehlende Anschlußfähigkeit an geltende nationale Normen oder kulturelle Bedeutungsgehalte als konstruktivistische Interpretation dessen zu behandeln, was eine „unbequeme internationale Regel“" ausmacht.

Hinzu kommt, dass sich politische und gesellschaftliche Akteure häufig als „Normunternehmer“ gerieren, die mittels strategischer sozialer Konstruktionen internationale Regeln mit den nationalen Normen kompatibel machen. Dies führt zu einer weiteren konstruktivistisch inspirierten Hypothese, welche die Varianz zwischen Regelanerkennung und -einhaltung erklären soll. Danach wird Regeleinhaltung umso wahrscheinlicher, je mehr es transnationalen oder gesellschaftlichen Akteuren - „Normunternehmern“ - gelingt, über strategische Konstruktionen und das „framing“ sozialer Probleme andere Akteure von der Anschlußfähigkeit der internationalen Regeln an nationale Verfahren zu überzeugen. Diese Hypothese bildet so etwas wie das konstruktivistische Gegenstück zur oben formulierten liberal-akteurszentrierten Annahme, wonach internationale Regeln und Institutionen zu einer Umverteilung von Ressourcen zwischen gesellschaftlichen und politischen Akteuren führen.

Zusammengefasst lassen sich vier Annahmen zum Problem der Regelbefolgung aus dem konstruktivistischen bzw. soziologischen Institutionalismus ableiten. Die Einhaltung unbequemer internationaler Regeln hängt ab von

1. der Einbeziehung der Regelzielgruppe in das Verfahren der Normfindung (Verfahrenslegitimitäts-Hypothese); 
2. der Einbettung in umfassendere und weithin als legitim bzw. für die Staatengemeinschaft als konstitutiv angesehene internationale Institutionen (institutionelle Legitimitäts-Hypothese);

3. der unmittelbaren Rechtswirkung und der innerstaatlichen Einklagbarkeit der Regel (rechtliche Internalisierungs-Hypothese);

4. der Überzeugungsfähigkeit transnationaler oder nationaler „Normunternehmer“ in den gesellschaftlichen Umfeldern (soziale Internalisierungs-Hypothese).

\section{4 Überlegungen zu einer Synthese von Rationalismus und Konstruktivismus}

Die oben entwickelten rationalistischen und konstruktivistischen Perspektiven zum Problem der Befolgung internationaler Regeln unterscheiden sich dadurch, dass sie zum einen den gemeinsamen Ausgangspunkt - „unbequeme internationale Normen“ - jeweils unterschiedlich konzeptualisieren. Für rationalistische Institutionalisten geht es in erster Linie um die mit der Regelbefolgung zusammenhängenden (materiellen und ideellen) Kosten. Konstruktivistische Institutionalisten betonen dagegen die fehlende Anschlußfähigkeit der internationalen Norm an nationale Regeln, Verfahren und kulturelle Bedeutungsgehalte. Zum anderen stellen beide Perspektiven auf jeweils unterschiedliche Mechanismen und Kausalpfade von der Normanerkennung zur Normeinhaltung ab. Diese Mechanismen wurden oben im einzelnen diskutiert und werden in Abbildung 1 zusammengefasst.

Bedeutet dies, daß die beiden institutionalistischen Perspektiven zur Frage der Regeleinhaltung sich wechselseitig ausschließen, weil sie erstens das Problem unterschiedlich beschreiben und zweitens verschiedene Mechanismen der Regeldurchsetzung betonen? Es ist in der Tat richtig, dass beide Perspektiven jeweils unterschiedliche Handlungslogiken betonen, die sich auf der theoretisch-analytischen Ebene wechselseitig ausschliessen. Die Logik der Angemessenheit widerspricht grundsätzlich zweckutilitaristischem Handeln und ist auch nicht mit verständigungsorientiertem Handeln vereinbar. Davon ist allerdings die empirisch-phänomenologische Ebene zu trennen. Die Spiele, die wirkliche Akteure in der wirklichen Welt spielen (Scharpf 1997), kombinieren meistens die metatheoretisch differenzierten Handlungslogiken der instrumentellen, normgeleiteten und kommunikativen Rationalität. Es kann damit für die Forschung nicht darum gehen, sich metatheoretisch für ausschließlich eine Handlungslogik zu entscheiden, sondern herauszufinden, welche Handlungslogik in welcher empirischen sozialen Situation dominiert. Dabei können rationalistische und konstruktivistische Ansätze als heuristische Mittel betrachtet werden, die den Blick auf jeweils unterschiedliche Elemente der Wirklichkeit richten bzw. verschiedene Erklärungsfaktoren der gleichen empirischen Wirklichkeit betrachten. 


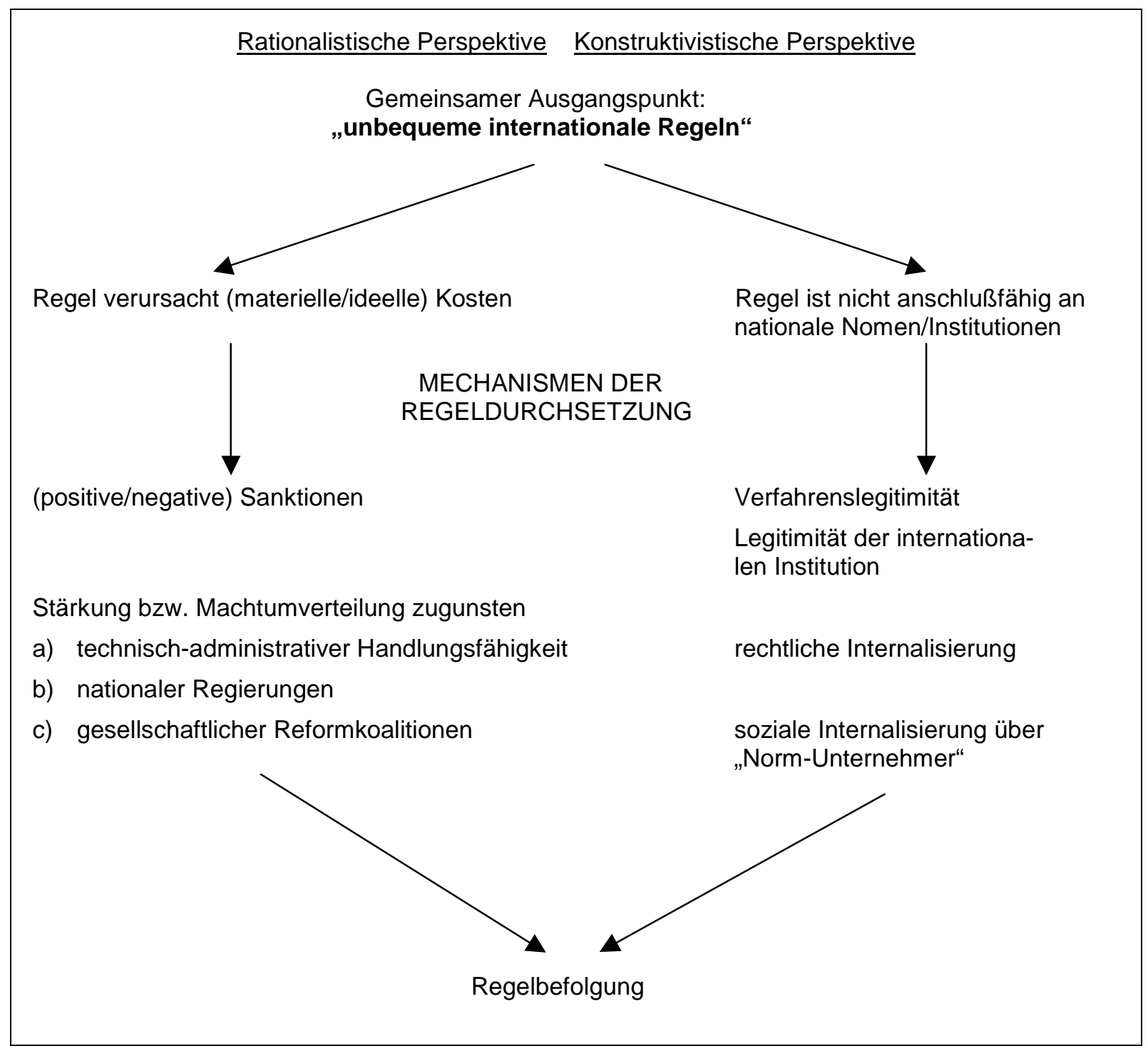

Abbildung 1: Rationalistische und konstruktivistische Perspektiven zum Problem der Regelbefolgung

Was bedeutet das für unsere Fragestellung? Zunächst ergibt sich, dass die unterschiedliche Konzeptualisierung einer „unbequemen Regel“ durch die beiden theoretischen Perspektiven nicht schon zu miteinander unvereinbaren Problembeschreibungen führen muss. Eine internationale Norm, die an nationale Institutionen und Bedeutungsgehalte nur wenig anschlussfähig ist, wird ebenfalls ideelle und in der Regel auch materielle Kosten bei der Umsetzung verursachen. Umgekehrt gilt, dass Akteure, die von den Kosten einer internationalen Regel betroffen sind, häufig dazu neigen, die fehlende Anschlussfähigkeit der Norm an nationale Bestimmungen zu etablieren.

Was die von uns identifizierten Mechanismen der Regeldurchsetzung angeht, so handelt es sich hier um teilweise funktional äquivalente kausale Pfade. Eine hohe Legitimität der internationalen Normen bzw. deren rechtliche Inernalisierung im nationalen System vermag zum Beispiel unzureichende Sanktionierungsmechanismen zu kompensieren (und umgekehrt). In Abwesenheit gesellschaftlicher Reformkoalitionen, deren Machtressourcen durch die internationale Norm ge- 
stärkt werden, können Normunternehmer über geschickte soziale Konstruktionen diskursive Überzeugungsprozesse einleiten, die zur Regelbefolgung führen.

Die Mechanismen der Regeldurchsetzung können sich aber auch wechselseitig verstärken. Wenn eine internationale Institution hohe Legitimität genießt, lassen sich weitreichende Überwachungs- und Sanktionsmechanismen politisch und gesellschaftlich eher durchsetzen. Umgekehrt gilt, dass der Druck internationaler Sanktionen oder allein schon die Drohung damit die Bereitschaft von Regierungen erhöhen kann, sich auf diskursive Argumentationsprozesse über das Maß der Regelbefolgung einzulassen. Innerhalb der gesellschaftlichen Umfelder können „NormUnternehmer“ durch Überzeugungsprozesse dazu beitragen, gesellschaftliche Mehrheiten zugunsten der internationalen Norm zu bilden.

Allerdings ist es auch möglich, dass sich die hier beschriebenen Mechanismen wechselseitig behindern. Wie die amerikanische Sanktionspolitik gegenüber dem Irak über einen Zeitraum von mehr als zehn Jahren gezeigt hat, führen harte Maßnahmen gegen Regelverletzungen nicht unbedingt zur Einhaltung der Normen, wenn diese von den Adressaten als illegitim wahrgenommen werden. Im Gegenteil, die Sanktionen verstärken noch die perzipierte Illegitimität der Norm und verhindern geradezu, dass sich Reformkoalitionen und Normunternehmer herausbilden, die zugunsten der internationalen Regeln mobilisieren.

Schließlich ist darauf hinzuweisen, dass die nachhaltige Befolgung unbequemer internationaler Regeln wahrscheinlich nur über deren Internalisierung in Sozialisationsprozessen zu erreichen ist. Da die Mechanismen, die auf instrumenteller Rationalität beruhen, die Interessen der Akteuren nicht verändern, werden unbequeme Regeln entsprechend nur so lange befolgt, wie die Sanktionen bei Vertragsverletzung aufrechterhalten werden oder solange die Reformkoalitionen von den Regeln profitieren. Sozialisation über Lern- und Überzeugungsprozesse, die zu rechtlicher und sozialer Internalisierung führen, verändert hingegen Interessen und Identitäten der Akteure, so dass positive oder negative Anreize zur Regeleinhaltung zunehmend unnötig werden.

\section{Empirische Illustrationen: Umweltpolitik in Europa und internationale Menschenrechte}

Wir können an dieser Stelle die von uns entwickelten Hypothesen nicht einer systematischen empirischen Überprüfung unterziehen. Wir beschränken uns deshalb darauf, mit Hilfe empirischer Beispiele aus dem Bereich der europäischen Umweltpolitik und den internationalen Menschenrechten die Plausibilität unserer Hypothesen zu illustrieren. Die Fallauswahl ergibt sich aus den unterschiedlichen Graden der Legalisierung und Legitimität der entsprechenden Normen und Instititutionen. Während sowohl europäische Umwelt- als auch internationale Menschenrechtsnormen Formen des Rechts jenseits des Nationalstaates bilden, so unterscheiden sie sich hinsichtlich ihres Rechtscharakters doch erheblich. Zwar gehören Menschenrechtsregime zu den internationalen Abkommen, deren Legalisierung im Sinne der Präzision der Normen und Regeln sowie der Überwachungs- und Sanktionierungsmechanismen (Abbott, et al. 2000) mit am weite- 
sten fortgeschritten ist. Anders als internationales Recht genießt europäisches (Umwelt)-Recht jedoch Vorrang vor nationalem Recht und ist in vielen Fällen unmittelbar anwendbar. Selbst völkerrechtlich verbindliche Menschenrechtsnormen bedürfen hingegen der Umsetzung und Internalisierung in das nationale Recht. Auch die Regelungsdichte ist in der europäischen Umweltpolitik ungleich höher als im Falle internationaler Menschenrechtsnormen. Zwar decken die verschiedenen internationalen Menschenrechtskonventionen den Bereich der politischen und bürgerlichen sowie der wirtschaftlichen und sozialen Rechte weitgehend ab. Die über 220 europäischen Rechtsakte enthalten jedoch sehr viel spezifischere und umfassendere Regelungen der verschiedenen Umweltmedien. Darüber hinaus verfügt die EU über ein ausdifferenziertes, der Kontrolle der Mitgliedsstaaten weitgehend entzogenes Sanktionssystem, das dem Europäischen Gerichtshof die Möglichkeit einräumt, beachtliche Geldbußen zu verhängen.

Schließlich unterscheiden sich europäische Umwelt- und internationale Menschenrechtsnormen hinsichtlich ihrer Legitimität. Einerseits haben sowohl Menschenrechts- als auch Umweltnormen konstitutiven Charakter für moderne rechtsstaatliche Demokratien. Liberale Demokratien sind über die Achtung der Menschenrechte definiert; in zunehmenden Maße stellt die Einhaltung der Menschenrechte ein Indikator dafür dar, ob Staaten zu den geachteten Mitgliedern der internationalen Gemeinschaft gehören. Was die Erhaltung der Umwelt angeht, so hat Umweltschutz in vielen Demokratien des Westens Verfassungsrang. Andererseits genießen europäische Umweltregelungen als Teil des Europarechts einen höheren Grad an Normanerkennung und damit Legitimität als die internationalen Menschenrechte. Während sich EU-Mitgliedstaaten häufig um die beste Problemlösungsphilosophie in der Umweltpolitik streiten, erkennen sie die Geltung europäischer Umweltnormen als Teil des EU-Rechtssystems von vorneherein an. Demgegenüber erfolgt Normanerkennung der internationalen Menschenrechte durch die Staaten keinesfalls automatisch, da sie die entsprechenden internationalen Abkommen jeweils unterzeichnen, ratifizieren und sich den Überprüfungs- und Sanktionierungsmechanismen unterwerfen müssen. Die Phase der Normanerkennung muss daher bei der Um- und Durchsetzung von internationalen Menschenrechtsnormen in nationales Recht gesondert untersucht werden, wohingegen sie im EU-Umweltrecht praktisch gegeben ist.

Im folgenden werden wir die insgesamt acht Hypothesen für die beiden Bereiche diskutieren. Der Vergleich soll zeigen, inwiefern die unterschiedlichen Charakteristika der beiden Politikfelder die Wirkungsweise rationalistischer bzw. konstruktivistischer compliance-Mechanismen begünstigen. 


\subsection{Wenn sich Mitgliedstaaten nicht an europäische Umweltnormen halten²}

\section{Ausgangspunkt: Unbequeme Regeln}

Die Normbildung in der europäischen Umweltpolitik lässt sich als ein „regulativer Wettbewerb” zwischen hoch regulierten Mitgliedstaaten begreifen, die versuchen, ihre anspruchsvollen Umweltstandards auf der europäischen Ebene zu harmonisieren, um dem transnationalen Charakter vieler Umweltprobleme gerecht $\mathrm{zu}$ werden und Wettbewerbsnachteile für ihre Industrien zu vermeiden (Héritier 1996, Héritier, et al. 1994). Da die Länder mit hohen Umweltstandards unterschiedliche Regulierungstraditionen haben, konkurrieren sie darum, ihren eigenen Regulierungsansatz zu europäisieren, um Anpassungskosten zu minimieren. Dieser regulative Wettbewerb führt nicht nur zu erheblichen Umsetzungskosten für umweltpolitische „Nachzügler”, die ihre Regulierungsstrukturen an die hohen Anforderungen der EU anpassen müssen. Selbst höher regulierte Länder sehen sich zu kostspieligen Anpassungen genötigt, wenn sie sich nicht auf europäischer Ebene mit ihrem Regulierungsansatz durchsetzen. So setzte sich Deutschland in den 80er Jahren bei der europäischen Luftreinhaltepolitik mit seinem dem Stand der Technik verpflichteten Emissionsansatz weitgehend durch. Großbritannien hatte hingegen das Nachsehen. Die britische Industrie musste deshalb nicht nur in teure Umwelttechnologien (z.B. Filteranlagen, einige davon ,made in Germany”) investieren. Die britische Verwaltung war gezwungen, ihren qualitätsorientierten Ansatz, der auf der Abwägung von ökologischem Nutzen und ökonomischen Kosten umweltpolitischer Maßnahmen beruhte, in der Luftreinhaltepolitik aufzugeben. Das Beispiel zeigt, dass Normen, die nicht anschlussfähig sind an nationale Regulierungstraditionen, umfangreiche materielle und immaterielle Kosten mit sich bringen können. Die Umweltinformationsrichtlinie von 1990 stieß hingegen in vielen Mitgliedstaaten auf massiven Widerstand, obwohl ihre Umsetzung und Anwendung nur begrenzte Kosten verursachte. Die Richtlinie verpflichtet Behörden zur Herausgabe von Umweltinformationen, die sie nur unter bestimmten Bedingungen verweigern können. Obwohl der befürchtete administrative Aufwand weitgehend ausblieb, haben viele Verwaltungen versucht, die Anwendung der Regeln z.B. durch hohe Bearbeitungsgebühren zu umgehen (Hallo 1996). Transparenz und offener Zugang zu Informationen sind mit der Verwaltungstradition vieler Länder, nach der die im Besitz der Behörden befindlichen Informationen grundsätzlich geheim sind, nur schwer vereinbar. In diesem Fall ging es also weniger um Kosten als um die fehlende Anschlussfähigkeit der Norm.

\section{Grad der Legalisierung und Sanktionen}

Wenn es nach den über 15.000 aktenkundigen Vertragsverletzungen geht, welche die Kommission in den letzten 30 Jahren gegen die Mitgliedstaaten eingeleitet hat, wirkt sich der Spezifikationsgrad einer Regel entscheidend auf deren Einhaltung aus. Über 90\% aller Verfahren beziehen sich auf die Verletzung von Richtlinien, die im wesentlichen Rahmen- und Zielvorgaben enthalten und erst von den Mitgliedstaaten ins nationale Recht umgesetzt werden müssen. Verordnungen hingegen, die relativ spezifische Vorgaben enthalten und unmittelbar anwendbar sind, ma-

\footnotetext{
${ }^{2}$ Zum folgenden vgl. ausführlich Börzel 2000 und Börzel i.E.
} 
chen nur einen geringen Prozentsatz aus (etwa 3\%). ${ }^{3}$ Dies ist um so bemerkenswerter, als es ungefähr dreimal so viele Verordnungen wie Richtlinien im Gemeinschaftsrecht gibt. Im Bereich der Umweltpolitik reguliert die EU fast ausschließlich über Richtlinien, die oft recht vage Formulierungen enthalten. Daraus ergeben sich für die Mitgliedstaaten nicht nur Unsicherheiten hinsichtlich der Regelanforderungen. Sie haben erheblichen Spielraum bei der Umsetzung, den sie nicht selten nutzen, um unbequeme Regelungen zu umgehen. So erlaubt die Umweltinformationsrichtlinie den Mitgliedstaaten, eine ,,angemessene“ Bearbeitungsgebühr für die Bereitstellung von Information zu erheben. Was jedoch als „,angemessen“ anzusehen ist, wird von der Richtlinie nicht spezifiziert, so dass deutsche Behörden z.B. den Stundenlohn eines leitenden Verwaltungsangestellten bei ihrer Gebührenberechnung zugrunde gelegt und für einige Aktenkopien über 100 Mark verlangt haben. Im Rahmen des Vertragsverletzungsverfahrens nach Art. 226 EG-Vertrag lassen sich sowohl Regelanforderungen klären als auch absichtliche Regelverstöße unterbinden. Dabei ist nur in wenigen Fällen ein Urteil des Europäischen Gerichtshofes von Nöten. Über zwei Drittel aller von der Kommission vorgebrachten Regelverletzungen werden in informellen Verhandlungen mit den nationalen Regierungen beigelegt. Die Aussicht auf ein EuGH-Verfahren fördert allerdings die Bereitschaft der Mitgliedstaaten, sich auf solche Vermittlungsprozesse einzulassen.

\section{Management-Hypothese}

Der regulative Wettbewerb in der europäischen Umweltpolitik führt zu erheblichen Kosten bei der Regelbefolgung, insbesondere in den Ländern mit niedrigen Umweltstandards, deren Handlungskapazitäten gewöhnlich auch schwächer ausgebildet sind als die der Umweltpioniere. Die Umsetzung der Abwasserrichtlinie hat in Spanien z.B. Investitionen von mehreren HundertMillionen Mark notwendig gemacht, weil nur etwa 40\% der Kommunen über Kläranlagen verfügen, welche die Anforderungen der Richtlinie erfüllen. Um den umweltpolitischen Nachzüglern bei der Bewältigung der Anpassungskosten zu helfen, stellt die Europäische Union besondere Finanzmittel bereit. So sind 50\% der Gelder aus dem Kohäsionsfond für die Umsetzung von Umweltprogrammen bestimmt. Außerdem gibt es verschiedene EU-Programme (MEDSPA, ENVIREG oder LIFE) sowie günstige Kredite der Europäischen Investitionsbank oder der Europäischen Bank für Wiederaufbau und Entwicklung, die finanzielle Hilfestellung leisten. Neben der Bereitstellung finanzieller Ressourcen fördern europäische Institutionen auch den Austausch von Informationen und technischer Expertise. Über die Umweltausschüsse der Versammlung der Regionen Europas und des Ausschusses der Regionen haben z.B. deutsche Abfallkataloge ihren Weg in einige Mittelmeerregionen gefunden, als diese nach Vorlagen für die Umsetzung europäischer Abfallrichtlinien suchten.

3 Die Informationen zu den Verletzungen europäischer Normen und Regeln stammen aus einer Datenbank, die unter der Leitung von Tanja Börzel z.Zt. am Europäischen Hochschulinstitut erstellt wird und sämtliche Vertragsverletzungen umfasst, welche die Kommission in den 30 letzten Jahren offiziell gegen die Mitgliedstaaten eingeleitet hat. Vgl. Börzel 2001. Siehe auch http://www.iue.it/rsc/rsc_tools. 


\section{Liberal-institutionenzentrierte Hypothese}

Die nationalen Regierungen verweisen gerne auf innenpolitische Vetospieler (Regionen, Gewerkschaften, Interessengruppen), wenn sie sich vor der Kommission für Verstöße gegen das Gemeinschaftsrecht rechtfertigen müssen. Die Verteilung der aktenkundigen Vertragsverletzungsverfahren lässt jedoch keinen Zusammenhang zwischen der politischen Autonomie staatlicher Akteure und dem offiziellem Regelbefolgungsgrad eines Landes erkennen. Die politischen Systeme von Portugal und Griechenland sind traditionell staatsdominiert und weisen eine vergleichsweise niedrige Zahl institutioneller Vetospieler auf. Trotzdem gehören sie zu den Staaten mit den meisten Vertragsverletzungsverfahren. Umgekehrt verfügen die Regierungen der Niederlande und Deutschlands aufgrund korporatistischer Strukturen, zu denen im Falle Deutschlands noch eine föderalistische Staatsgliederung hinzukommt, über weitaus weniger Handlungsautonomie, sehen sich aber einer geringeren Zahl an Vertragsverletzungsverfahren gegenüber.

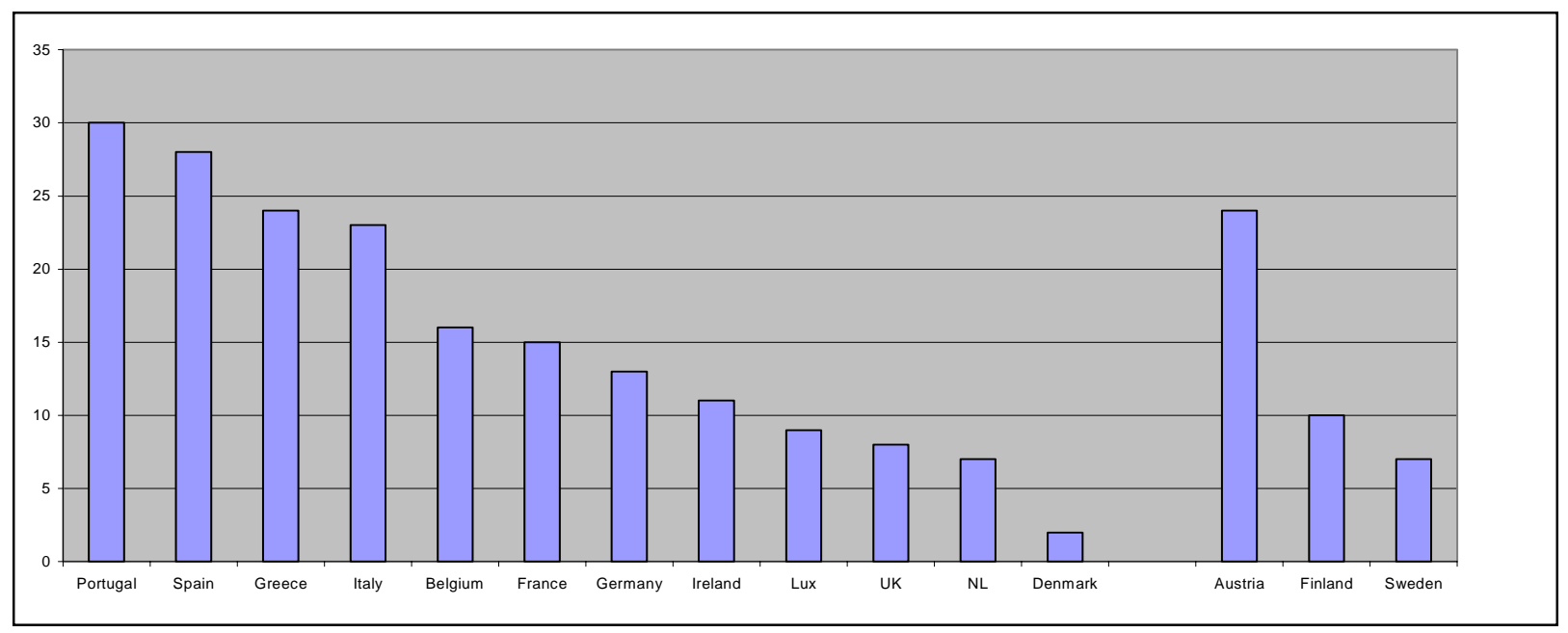

Quelle: Datenbank zur Einhaltung von Gemeinschaftsrecht, EHI, Robert Schuman Centre

\section{Abbildung 2: Durchschnittliche Zahl der begründeten Stellungnahmen pro Jahr im Umwelt- bereich für die einzelnen Mitgliedstaaten 1971-1999}

Ein Problem dieser institutionenzentrierten Perspektive ist allerdings, dass sie die Rolle faktischer Vetospieler vernachlässigt, die zwar nicht über institutionelle Blockademöglichkeiten verfügen, aber trotzdem einen erheblichen Einfluss auf den Politikprozess ausüben können. Dies zeigt sich z.B. bei der Umsetzung der europäischen Umweltpolitik in Frankreich, wo weder mächtige Regionen noch korporatistisch verfasste Interessengruppen formal den Prozess behindern können. Die informelle Verwaltungspraxis einer flexiblen und mit den Betroffenen ausgehandelten Anwendung strikter nationaler Standards auf der lokalen Ebene bildet nicht nur ein Gegengewicht zum zentralisierten politischen Entscheidungsprozess (Héritier, et al. 1994, 144147), er hat Frankreich zahlreiche Vertragsverletzungsverfahren eingehandelt, z.B. im Bereich der Umweltverträglichkeitsprüfung. 


\section{Liberal-akteurszentrierte Hypothese}

In der europäischen Umweltpolitik lassen sich zahlreiche Beispiele finden, wo eine Richtlinie zu einer Machtumverteilung in den Mitgliedstaaten geführt hat - sowohl zugunsten einer reformfreudigen Regierung als auch zugunsten gesellschaftlicher Reformkoalitionen. So gibt es im Bundesumweltministerium durchaus Kräfte, die integrative und partizipative, also mit der deutschen Regulierungstradition nur schwer vereinbare Politikinstrumente wie die Umweltverträglichkeitsprüfung (UVP) oder das integrierte Genehmigungsverfahren für Industrieanlagen (IVU) unterstützen, weil sie darin eine Möglichkeit sehen, das deutsche Umweltrecht zu modernisieren. Sie haben die Formulierung dieser Richtlinien auf europäischer Ebene zumindest informell unterstützt und versucht, innenpolitische Gegner (Vollzugsbehörden, Industrieverbände) mit einer bereits verabschiedeten Richtlinie, die ins nationale Recht umgesetzt werden muss, vor vollendete Tatsachen zu stellen. Diese Strategie der Selbstbindung, um innenpolitische Reformwiderstände zu brechen, ist allerdings nur bedingt aufgegangen. Die vollständige Umsetzung der UVP-Richtlinie von 1985 dauerte mehrere Jahre und bedurfte zweier Verfahren vor dem Europäischen Gerichtshof, die auf Beschwerden von Umweltgruppen zurückgingen. Die Umsetzung der IVU-Richtlinie von 1996 sowie der 1997 novellierten UVP-Richtlinie sollte durch ein Umweltgesetzbuch erfolgen, das die stark sektoralisierte Gesetzgebung zu den einzelnen Umweltmedien zusammenfassen und integrieren würde. Der von einer Expertenkommission 1998 vorgelegte Entwurf ist jedoch am breiten Widerstand aus den Ländern, Kommunen und der Industrie gescheitert. Deutschland droht jetzt ein Vertragsverletzungsverfahren wegen Nichtumsetzung der Richtlinien. Innenpolitische Reformkräfte erfahren eine weitere Stärkung durch die Überwachungs- und Sanktionsmechanismen der EU. Aufgrund ihrer begrenzten Ressourcen ist die Kommission nicht in der Lage, die Einhaltung des Gemeinschaftsrechts lückenlos zu überwachen. Sie ist bei der Aufdeckung von Regelverstößen auf die Beschwerden von Bürgern und Unternehmen angewiesen. Dies gibt gesellschaftlichen Reformkoalitionen ein wirksames Mittel an die Hand, um Druck sowohl auf ihre Regierung als auch auf Reformgegner auszuüben, sich den Anpassungskosten unbequemer Regeln zu stellen. Der vereinte Druck von „unten“ - durch die politische Mobilisierung von Unweltgruppen - und „oben“ - durch die (von den Umweltgruppen alarmierte) Kommission hat sich bei der Durchsetzung unbequemer Normen wie der Umweltinformationsrichtlinie als recht wirksam erwiesen. Die umfassende Partizipation gesellschaftlicher Akteure zumindest bei der Umsetzung europäischer Umweltpolitik ist seit Anfang der 90er Jahre erklärtes Ziel der Kommission (Commission of the European Communities 1991). Verfahrensregelungen wie die UVP oder die Umweltinformationsrichtlinie, die den Bürgern Informationsund Mitwirkungsrechte z.B. bei Genehmigungsverfahren einräumen, sollen zu einer verbesserten Überwachung und Durchsetzung von (EU-) Umweltstandards führen. In Spanien, wo Umweltgruppen und Bürgerinitiativen traditionell über wenig politischen Einfluss verfügen, haben diese neuen Partizipationsrechte tatsächlich zu einer Stärkung gesellschaftlicher Kräfte in der Umweltpolitik geführt, z.B. wenn es um Genehmigungsverfahren von Bau- und Industrievorhaben geht. 


\section{Verfahrenslegitimität}

Die fehlende Einbeziehung innenpolitischer Akteure, die für die Einhaltung europäischer Normen und Regeln entscheidend sind, in den Normenbildungsprozess kann zu schwerwiegenden Problemen bei der Umsetzung führen. Dies gilt nicht nur für die Zielgruppen einer Regel, sondern auch für ihre Adressaten. Der weitgehende Ausschluss der Fachverwaltungen und Vollzugsbehörden aus dem europäischen Entscheidungsprozess zur Umweltverträglichkeitsrichtlinie oder zur Umweltinformationsrichtlinie hat den Reformkräften im Bundesumweltministerium zwar ermöglicht, die Richtlinien im Rat auf den Weg zu bringen. Im Umsetzungsprozess sind die beiden Richtlinien aber auf massiven Widerstand getroffen, da sie an die deutsche Verwaltungstradition kaum anschlussfähig sind (siehe oben). Angesichts der Führungsrolle, die Deutschland lange Zeit in der Umweltpolitik innehatte und nach Ansicht vieler Beamten immer noch innehat, erfahren europäische Regelungen, die mit dem deutschen Regulierungsansatz nicht vereinbar sind, wenig Akzeptanz. Dabei führen Verwaltungsbeamte nicht nur die vergleichsweise hohe Problemlösungsfähigkeit deutscher Umweltpolitik ins Feld, sondern verweisen auch auf die Kohärenz des deutschen Rechtssystems, die durch die Übernahme „regulierungsfremder“ Elemente wie der medienübergreifenden Evaluierung gefährdet werde.

\section{Institutionelle Legitimität}

An der mangelnden Folgebereitschaft bei „unbequemen“ EU-Umweltnormen ändert auch die Tatsache nichts, dass die EU im allgemeinen hohe Legitimität in der deutschen Verwaltung genießt. Weder die Notwendigkeit europäischer Umweltgesetze noch ihr Geltungsvorrang im nationalen Recht wird in Frage gestellt. Es geht vielmehr um bestimme Regelungen, die als mit den historisch gewachsenen deutschen Regulierungsstrukturen und Verwaltungstraditionen nicht vereinbar gelten. Nicht zuletzt deshalb fordern die deutschen Länder eine strikte Anwendung des Subsidiaritätsprinzips, das die Rolle der EU auf die Setzung umweltpolitische Vorgaben reduzieren soll und den Mitgliedsstaaten größtmöglichen Spielraum bei der Umsetzung lässt. Die Verteilung der Vertragsverletzungsverfahren deutet ebenfalls daraufhin, dass kein monokausaler Zusammenhang zwischen der allgemeinen Unterstützung für die EU einerseits und der Einhaltung ihrer Umweltgesetze in den Mitgliedsstaaten besteht. Die größten Umweltsündern (Italien, Belgien, Griechenland) gehören zu den europafreundlichsten Ländern, während die Dänen als umweltpolitische Musterknaben gleichzeitig zu den größten Europaskeptikern zählen. Das umweltpolitische Selbstverständnis in den Mitgliedstaaten und damit die Legitimität, die Umweltnormen als solche genießen, scheinen von größerer Relevanz für das Maß der Normeinhaltung zu sein. Einerseits lässt sich Deutschland ungern von der EU sagen, wie effektive Umweltregulierung gemacht wird. Andererseits ist es nur schwer mit dem deutschen Anspruch vereinbar, eine umweltpolitische Führungsrolle in Europa zu spielen, wenn Deutschland mehr Verfahren beim Europäischen Gerichtshof wegen Verletzungen von EU-Umweltrecht anhängig hat, als umweltpolitische Nachzügler wie Spanien oder Portugal. Gleichzeitig erweisen sich europäische Ressourcentransfers als wichtiger Faktor, der nicht nur Handlungskapazitäten nationaler Regierungen, sondern auch die Akzeptanz europäischer Normen und Regeln befördert. Die Bereit- 
schaft zur Internalisierung neuer Problemlösungsansätze in der Umweltpolitik steigt, wenn sich die damit verbundenen Kosten reduzieren. Solange in Spanien, Portugal oder Griechenland der Industrie keine Investitionshilfe beim Erwerb umweltfreundlicher Technologien geleistet wird, überrascht es kaum, dass diese Länder den „Stand der Technik” beim Emissionsschutz als „typisch nordisches Konzept" ablehnen, das für den Süden nicht taugt.

\section{Rechtliche Internalisierung}

Auf die Partizipation gesellschaftlicher Akteure in der europäischen Umweltpolitik als Faktor, der die Normeinhaltung befördert, wurde bereits im Zusammenhang mit der liberalakteurszentrierten Hypothese hingewiesen. Um den Druck zu erhöhen, schlagen Bürger und Umweltgruppen häufig den Rechtsweg ein, wenn sich nationale Behörden z.B. weigern, die partizipativen Instrumente der europäischen Umweltpolitik zur Anwendung zu bringen (Knill und Lenschow 2000). Die Mobilisierung gesellschaftlicher Kräfte trägt so entscheidend zur rechtlichen Internalisierung europäischer Umweltnormen bei. Bei der Klärung ihres Bedeutungsgehalts und Geltungsbereichs spielen nicht nur nationale Gerichte eine wichtige Rolle, sondern auch der Europäische Gerichtshof, der von der Kommission aufgrund von Beschwerden nationaler Umweltgruppen oder Bürger(initiativen) bzw. von nationalen Gerichten im Rahmen des Vorlageverfahrens nach Art. 234 (ex-Art. 177) EG-Vertrag angerufen wird. Die Bereitschaft nationaler Gerichte, einen Rechtsdiskurs über europäische Umweltnormen zu führen, variiert allerdings beträchtlich, sowohl zwischen den Mitgliedsstaaten als auch zwischen den verschiedenen Gerichten innerhalb eines Mitgliedsstaaates (Conant 2001). Auch mündet der Rechtsdiskurs nicht notwendigerweise in die Normeinhaltung. So haben deutsche Verwaltungsgerichte in ihrer Rechtsprechung die restriktive Auslegung und Anwendung der europäischen Umweltverträglichkeitsprüfungsrichtlinie durch die deutschen Behörden gestützt.

\section{Soziale Internalisierung}

Während gesellschaftliche Akteure die Kosten der Normeinhaltung bzw. Nichtbefolgung maßgeblich beeinflussen können, indem sie - oft gemeinsam mit der Kommission - Druck auf die Adressaten und Zielgruppen europäischer Umweltregelungen ausüben, treten sie als „Normunternehmer" und Sozialisationsagenten, die soziale Lern- und Überzeugungsprozesse anstoßen, weniger in Erscheinung. Der technisch-rechtliche Charakter von Umweltregelungen macht es Umweltgruppen oft schwer, politische Entscheidungsträger und Umweltverwaltungen in einen öffentlichen Diskurs über die Legitimität der integrierten Vermeidung und Überwachung von Umweltverschmutzung oder des Umwelt-Audit und Management Systems zu verwickeln. Solche Auseinandersetzungen finden vorwiegend in wissenschaftlichen Foren wie juristischen und umweltpolitischen Fachzeitschriften oder Fachkongressen statt. Die Rolle von „Normunternehmern“ und ihr Einfluss auf die Einhaltung europäischen Umweltrechts ist bisher allerdings kaum systematisch untersucht worden. 
Die empirischen Beispiele aus der europäischen Umweltpolitik illustrieren die Plausiblität der aus rationalistischen und konstruktivistischen Ansätzen zur Institutionenwirkung abgeleiteten Hypothesen. Dabei zeigt sich, dass sich die verschiedenen Hypothesen keinesfalls gegenseitig ausschließen, sondern in unterschiedlichen Beziehungen zueinander stehen können. So mögen europäische Umweltnormen reformfreudige Regierungen gegenüber innenpolitischen Vetospielern stärken; gleichzeitig kann die fehlende Einbeziehung potentieller Reformgegner in den Entscheidungsprozess die Akzeptanz europäischer Normen auf der nationalen Ebene erheblich beeinträchtigen. Compliance-Mechanismen können sich aber auch wechselseitig verstärken, wenn z.B. die Moblisierung gesellschaftlicher Kräfte nicht nur zu externen Sanktionen führen, sondern auch zu gerichtlichen Auseinandersetzungen, die zur rechtliche Internalisierung einer Norm führen. Und der Aufbau von Handlungskapazitäten durch Ressourcentransfers vermag die Akzeptanz unbequemer Regeln zu fördern.

\subsection{Internationale Menschenrechte: Norminternalisierung und transnationale Netzwerke}

\section{Menschenrechte als „unbequeme Normen“}

Im folgenden berichten wir von den Ergebnissen eines Forschungsprojektes, das die Durchsetzung von Freiheits- und Persönlichkeitsrechten (u.a. Folterverbot) in elf Ländern des Südens (Afrika, Asien, Lateinamerika) sowie im früheren kommunistischen Osteuropa untersucht hat (Risse et al. 1999; i.E.). In allen elf Ländern wurden grundlegende Menschenrechte systematisch verletzt; bei unseren Untersuchungen ging es darum, die Bedingungen herauszufinden, unter denen es zu einer Verbesserung der Menschenrechtssituation kommt. Dazu wurde ein FünfPhasen „Spiralmodell“ entwickelt, das mit der systematischen Missachtung der Menschenrechte und dem Leugnen der Gültigkeit internationaler Normen in diesem Bereich beginnt. Im Unterschied zur europäischen Umweltpolitik ist in diesem Fall die Normgeltung selbst zunächst umstritten. Dass die in den diversen Menschenrechtskonventionen verankerten Normen für repressive Regime unbequem sind, ergibt sich dabei aus der Natur der Sache. Erst in der Phase taktischer Konzessionen, in der die Regime unter zunehmenden Druck seitens ihrer internationalen Umwelt und der innenpolitischen Opposition geraten, beginnen repressive Regierungen, sich allmählich auf die Sprache der Menschenrechte einzulassen. Von Normanerkennung kann allerdings erst dann gesprochen werden, wenn Regierungen die entsprechenden internationalen Menschenrechtsabkommen ratifizieren und sich ihren Überwachungsmechanismen unterwerfen, die Normen in nationales Recht umsetzen und ihren öffentlichen Diskurs im Sinne der Menschenrechte verändern. Auch hier zeigt sich, dass es unter Umständen ein weiter Weg ist von der Normanerkennung zur dauerhaften Einhaltung der wichtigsten Menschenrechte (vgl. dazu auch Liese 2001). 


\section{Grad der Legalisierung und Sanktionen}

Im Menschenrechtsbereich lässt sich die Legalisierungshypothese kaum bestätigen. Gerade die Freiheits- und Persönlichkeitsrechte gehören im Unterschied etwa zu sozialen und ökonomischen Rechten zu den Menschenrechten, die in den verschiedenen internationalen Abkommen am genauesten und am detailliertesten spezifiziert sind. Das gilt auch für die Überwachungs- und Kontrollverfahren. Trotzdem wird in mehr als 100 Ländern der Erde nach wie vor gefoltert, wie sich aus jedem Jahresbericht von Amnesty International entnehmen lässt. Eine genauere Überprüfung der Legalisierungshypothese in bezug auf Menschenrechte in Lateinamerika erbrachte ebenfalls einen negativen Befund (Lutz und Sikkink 2000). Für die von uns untersuchten Länder gilt ausserdem, dass die meisten zunächst gar nicht Vertragspartner der entsprechenden internationalen Abkommen waren.

Was positive Anreize oder negative Sanktionen angeht, so ergibt sich ein widersprüchlicher Befund. Materielle Sanktionen der internationalen Staatengemeinschaft gegen menschenrechtsverletzende Staaten werden zwar zunehmend und konsistent verhängt, wie eine quantitativvergleichende Untersuchung der Sanktionspolitiken der USA und der EU für die neunziger Jahre bestätigen konnte (Hazelzet 2001). Ihre Wirkungen hängen aber entscheidend von Randbedingungen ab, näherhin dem Ausmaß der sozialen Mobilisierung im Innern der menschenrechtsverletzenden Staaten. Wenn die gesellschaftliche Opposition gegen die repressiven Regime nur schwach entwickelt ist, dann bleiben internationale Sanktionen oft wirkungslos, selbst wenn sie über Jahre hinweg verhängt werden. Wirtschaftliche Sanktionen allein sind selten geeignet, den Menschenrechten zum Durchbruch zu verhelfen, wie zahlreiche Studien immer wieder bestätigt haben (Drezner 1999, 2000; Elliott 1998; Hufbauer, et al. 1990). Die Beispiele Irak, Syrien oder Libyen sprechen hier für sich. Schlimmstenfalls benutzen Diktaturen internationale Sanktionen sogar zur Stärkung ihrer innenpolitischen Machtposition; es kommt zu einer nationalistischen Gegenreaktion.

Umgekehrt gilt jedoch, dass das Anprangern menschenrechtsverletzender Regierungen vor der internationalen Öffentlichkeit selbst einer (ideellen) Sanktion ähnelt, insofern den meisten Regimen ihr Image in der Welt nicht gleichgültig ist. Nur wenige Regierungen lassen sich gern zu Schurkenstaaten erklären, zumal dieser Status meist mit handfesten materiellen Kosten verbunden ist. Zugleich können auch materielle Sanktionen durchaus positive Wirkungen zeigen, wenn sie von einer bereits voll mobilisierten Opposition dazu genutzt werden können, das Regime weiter unter Druck zu setzen. Dies war zum Beispiel in Kenia und Indonesien Anfang der neunziger Jahre der Fall (vgl. Jetschke 2000; Schmitz 1999). In diesen Fällen gelang es der gesellschaftlichen Opposition, international isolierte Regierungen zu Konzessionen zugunsten der Menschenrechte zu bewegen.

\section{Management-Hypothese}

Im Menschenrechtsbereich spielt die politisch-administrative Handlungskapazität der betroffenen Staaten zunächst keine Rolle. Schließlich geht es hier nicht um ungewollte Regelverletzun- 
gen, sondern um beabsichtigte Normverletzungen zur Herrschaftssicherung der repressiven Regime. Erst in späteren Phasen der Menschenrechtsdurchsetzung kommen die Vorgaben des „managed compliance“-Ansatzes zum Tragen, beim Übergang von der Normankennung zur Normeinhaltung. Vor allem in den Ländern des Südens, in der die Durchsetzung der Menschenrechte mit einem Machtwechsel verbunden war, dauerte es einige Zeit, bis die neuen liberalen Eliten ihre Machtbasis konsolidiert und die Kontrolle über den Staatsapparat übernommen hatten. Dabei kam es zu Menschenrechtsverletzungen seitens der Sicherheitskräfte, obwohl die Regierung sich den internationalen Normen verpflichtet hatte. Gleichzeitig fehlen vielen der ärmsten Länder der Erde (beispielsweise Uganda, vgl. Schmitz 1999) die Handlungskapazitäten, um die Polizei und das Militär in Richtung auf menschenrechtskonformes Verhalten auszubilden und die entsprechenden Verhaltensänderungen zu trainieren. In vielen Ländern boten Amnesty International und andere NGOs Hilfestellungen, um die entsprechenden staatlichen Handlungskapazitäten aufzubauen. Schließlich ist auf das zunehmende Problem der Menschenrechtsverletzungen durch nicht-staatliche oder para-staatliche Akteure (Guerillas, paramilitärische Kräfte, marodierende Banden etc.) hinzuweisen, das vor allem jene Länder des Südens betrifft, in denen staatliche Strukturen nur noch rudimentär vorhanden sind.

\section{Liberal-institutionenzentrierte Hypothese}

Diese Annahme ist für den Menschenrechtsbereich zu falsifizieren. Es geht bei der Durchsetzung der Menschenrechte gerade nicht darum, die staatliche Autonomie gegenüber den gesellschaftlichen Umfeldern zu stärken. Im Gegenteil, Normeinhaltung bedeutet hier die Kontrolle und Einhegung staatlicher Herrschaft.

\section{Liberal-akteurszentrierte Hypothese}

Der in dieser Hypothese angedeutete Wirkungszusammenhang erweist sich im Menschenrechtsbereich als höchst aussagekräftig. In allen von uns untersuchten Ländern war internationaler Druck auf menschenrechtsverletzende Staaten allein nicht ausreichend zur Beförderung der Normeinhaltung. Ohne soziale Mobilisierung ,,von unten“, also in den gesellschaftlichen Umfeldern der betroffenen Staaten, kam es nicht zu einer dauerhaften Verbesserung der Menschenrechtssituation. Eine der wichtigsten Wirkungen der internationalen Menschenrechtsnormen einerseits und der Arbeit transnationaler Menschenrechtsnetzwerke aus (I)NGOs, internationalen Organisationen und einzelnen - zumeist westlichen - Staatenvertretern andererseits (dazu Keck und Sikkink 1998) besteht darin, die gesellschaftliche Opposition gegen repressive Regime zu stärken. Menschenrechte und die Aktivitäten der transnationalen Netzwerke verändern also tendenziell die innenpolitischen Kräfteverhältnisse zugunsten der liberalen Opposition gegen autoritäre Regime.

In den Phasen der ungebrochenen Repression und des Leugnens internationaler Menschenrechtsnormen durch die Regierungen sind es vor allem die transnationalen Netzwerke, die Informationen über die Lage im Land verbreiten und die internationale Öffentlichkeit mobilisieren. Je mehr 
Regierungen sich gezwungen fühlen, taktische Konzessionen im Menschenrechtsbereich zu machen und je mehr sie die Kontrolle über den Menschenrechtsdiskurs verlieren, desto größer wird der Handlungsspielraum der innergesellschaftlichen Opposition. Gleichzeitig wächst ihre Vernetzung mit den transnational operierende Gruppen, die ihr zum einen materielle und informationelle Ressourcen zur Verfügung stellen und sie zum anderen über die Aufmerksamkeit der Weltöffentlichkeit vor der Repression schützen. Die entscheidende Wirkung taktischer Konzessionen besteht also nicht in erster Linie in einer dauerhaften Verbesserung der Menschenrechtssituation, sondern in der Mobilisierung im Innern des repressiven Staates. Viele Staatsführungen beginnen in dieser Phase einen Prozess der kontrollierten Liberalisierung, in deren Verlauf sich die herrschenden Eliten häufig über das Ausmaß der nötigen Reformen zerstreiten (O'Donnell und Schmitter 1986, 15-21).

Der hier angedeutete Wirkungszusammenhang lässt sich allerdings nicht ausschließlich auf der „rationalistischen“ Seite der zugrundeliegenden Handlungslogiken (vgl. Abbildung 1) verorten. Erstens geht es um das Zusammenspiel zwischen transnationalen „Normunternehmern“ (s.u. ausführlich) und Oppositionsgruppen in den gesellschaftlichen Umfeldern der repressiven Staaten. Zweitens geht die Stärkung der innenpolitischen Opposition im allgemeinen einher mit einer Sozialisation in internationale Menschenrechtsnormen und damit einer Präferenzänderung der gesellschaftlichen Gruppen. Der Fall Indonesien belegt, dass die Einigung der Opposition auf den Menschenrechtsdiskurs eine notwendige Voraussetzung für innenpolitischen Wandel darstellt (Jetschke 2000). Internationale Menschenrechtsnormen stellen Oppositionsgruppen im Innern der Länder nicht nur ideelle Ressourcen zur Durchsetzung ihrer Interessen zur Verfügung, sie wirken auf deren Präferenzen und Identitäten selbst ein.

\section{Verfahrenslegitimität}

Die Annahme, dass die Einbeziehung der Regelzielgruppe in das Verfahren der Normbildung die Bereitschaft zur Normeinhaltung erhöht, ist für den Bereich der Menschenrechte entweder nicht anwendbar oder sogar fragwürdig. Die meisten der hier untersuchten repressiven Regime waren nicht an den Verhandlungen über die entsprechenden Menschenrechtskonventionen beteiligt, sondern traten erst später den Abkommen bei. Generell gilt, dass die meisten internationalen Menschenrechtsabkommen von denjenigen staatlichen und nicht-staatlichen Akteuren gefördert und verhandelt wurden, die die entsprechenden Normen bereits innerstaatlich implementiert hatten und internationalisieren wollten. Lediglich Moravcsik argumentiert, dass das europäische Menschenrechtsregime vor allem aufgrund der Initiative von sich demokratisierenden Staaten im Sinne einer Selbst-Bindung an internationale Normen zustandekam (Moravcsik 2000). Dies könnte die Hypothese zur Verfahrenslegitimität stützen. Allerdings zeigt eine Untersuchung von demokratischen Ländern (Großbritannien und Israel), denen ein Verstoß gegen das Folterverbot vorgewurfen wurde, dass auch die Beteiligung an den Vertragsverhandlungen - in diesem Fall der Anti-Folterkonvention - keine automatische Normeinhaltung garantiert (Liese 2001). 


\section{Institutionelle Legitimität}

Die Annahme, dass die Normeinhaltung zunimmt, wenn die Regeln zu einer für die Staatenwelt konstitutiven internationalen Institution gehören, kann für den Menschenrechtsbereich bestätigt werden. In der Tat gehören die grundlegenden Menschenrechte heute zu einem Bereich internationaler Normen mit außerordentlich hoher institutioneller Legitimität. Wer heute ein anerkanntes Mitglied der internationalen Staatengemeinschaft sein will, muss dem Menschenrechtsregime beigetreten sein und sich an die Normen halten. Insofern sind die Menschenrechte zunehmend konstitutiv für die Weltgesellschaft; die dementsprechende Einschränkung nationaler Souveränitätsrechte ist kaum noch umstritten.

Für den Prozess von der Normanerkennung zur Normeinhaltung hat das die konkrete Folge, dass es zu einer Beschleunigung der Menschenrechtsdurchsetzung in den Fällen kommt, in denen die sonstigen Bedingungen für die dauerhafte Implementation der Menschenrechte gegeben sind (vor allem transnationale und innergesellschaftliche Mobilisierung). Dauerte es in den Fällen Südafrikas, Indonesiens und Chiles noch Jahrzehnte von der Phase der Repression bis zur Durchsetzung der Menschenrechte, so hat sich dieser Prozess in den neunziger Jahren in vielen Fällen erheblich beschleunigt - ein Beweis für die inzwischen hohe institutionelle Legitimität der Menschenrechtsnormen.

\section{Rechtliche Internalisierung}

Rechtliche Internalisierung ist in der Tat eine notwendige, wenn auch nicht hinreichende Bedingung für die dauerhafte Einhaltung der Menschenrechte. Ohne Rechtsstaatlichkeit keine dauerhafte Verwirklichung der Menschenrechte! In allen von uns untersuchten Fällen war die Ratifizierung internationaler Menschenrechtsabkommen (als Indikator für die Normanerkennung) nur dann mit einer Verbesserung der Menschenrechtssituation verbunden, wenn ihr die konkrete Umsetzung in nationales Recht folgte. Dabei ging es weniger um die Aufnahme eines Grundrechtekatalogs in die nationale Verfassung. Vielmehr erwies sich die Einrichtung unabhängiger nationaler Instanzen im Menschenrechtsbereich mit der Möglichkeit zur Individualbeschwerde sei es über die Institutionalisierung einer Ombudsperson, sei es über die Einsetzung nationaler Menschenrechtskommissionen - als unabdingbare Voraussetzung einer späteren nachhaltigen Verbesserung der Regeleinhaltung. Rechtliche Internalisierung im Sinne der Einklagbarkeit der Menschenrechte vor unabhängigen nationalen Instanzen einerseits und einer Veränderung des innenpolitischen Diskurses andererseits war eine der wichtigsten Bedingungen für eine spätere nachhaltige Regelbefolgung.

\section{Soziale Internalisierung über transnationale „Norm-Unternehmer“}

Untersuchungen bestätigen die herausragende Bedeutung transnationaler Menschenrechtsnetzwerke aus individuellen und kollektiven Akteuren, die verbunden sind durch gemeinsame Werte, einen gemeinsamen Diskurs und den engen Austausch von Informationen und anderen Ressourcen (Keck und Sikkink 1998). Solche Netzwerke vereinigen verschiedene Gruppen sozialer Ak- 
teure (politische Stiftungen, Kirchen, Gewerkschaften, Medien etc.), die Aktionen initiieren, anderweitig schwer verfügbare Informationen liefern und staatliche Akteure dazu drängen, ihre Menschenrechtspraxis zu rechtfertigen. Einheimische und internationale Nichtregierungsorganisationen (NGOs) bilden das dauerhafte Rückgrat dieser Netzwerke.

Im Prozess der Menschenrechtsdurchsetzung wirken transnationale Netzwerke als Sozialisationsagenten. Erstens sammeln und veröffentlichen Amnesty International, Human Rights Watch und andere NGOs Informationen über Menschenrechtsverletzungen in aller Welt und stellen sie der Weltöffentlichkeit, nationalen Regierungen sowie internationalen Organisationen zur Verfügung. Transnationale Netzwerke mobilisieren die internationale Öffentlichkeit, westliche Regierungen und Geberländer sowie internationale Organisationen, Menschenrechtsverletzungen in Ländern des Südens nicht tatenlos hinzunehmen. Die Sozialisationswirkung bezieht sich darauf, dass westliche Öffentlichkeiten und liberale Demokratien an ihre eigene Identitäten erinnert und aufgefordert werden, ihren (Menschenrechts-) Worten Taten, beispielsweise durch eine prinzipiengeleitete Aussenpolitik folgen zu lassen. Mangels materieller Ressourcen vertrauen transnationale Netzwerke hier auf die Kraft des besseren Arguments, auf die Überzeugungskraft des moralischen Protests gepaart mit Sachwissen und Expertise.

Zweitens wenden sich transnationale Menschenrechtsnetzwerke direkt an die repressiven Regime und verwickeln sie in eine öffentliche Auseinandersetzung, zunächst über die Normanerkennung, dann über die Normeinhaltung. Zu Beginn der Auseinandersetzung tendieren menschenrechtsverletzende Regierungen dazu, die Legitimität der internationalen Normen selbst abzulehnen, zumeist mit dem Verweis auf die nationale Souveränität. Unter wachsendem Druck der (internationalen) Öffentlichkeit und in Reaktion auf zunehmende internationale politische und wirtschaftliche Sanktionen kommt es später zu taktischen Konzessionen, zu denen auch die - zunächst rein verbale - Anerkennung internationaler Menschenrechtsnormen gehören. Die Auseinandersetzung zwischen transnationalen Netzwerken und Regierungen verändert sich nun zunehmend von einem Streit über die Legitimität der Normen zu einer Kontroverse über deren praktischen Einhaltung. Die Argumente werden immer legalistischer und konkreter, gleichzeitig nehmen sich beide Seiten immer stärker als gleichberechtigte Teilnehmer am Diskurs wahr. Mit anderen Worten, die argumentative Auseinandersetzung zwischen NGOs einerseits und normverletzenden Regierungen andererseits verändert sich im Laufe der Zeit von Polemik und wechselseitiger Beschimpfung zu einem durchaus ernsthaftem Dialog über die Einhaltung der Menschenrechte. Dabei folgt dieser Dialog im Laufe der Zeit mehr und mehr den Regeln verständigungsorientierten Handelns, das Verhalten beider Seiten ähnelt einem Prozess ,,argumentativer Selbstverstrickung“ (vgl. Risse 1999, Risse et al. i.E.).

Der Kommunikationsprozess entspricht dem oben beschriebenen rhetorischen Handeln vor Gericht, wobei die transnationalen Netzwerke die Rolle der Ankläger spielen und die normverletzenden Regierungen sich verteidigen. Weil beide Seiten eine internationale und zunehmend auch innenpolitische Öffentlichkeit überzeugen müssen und weil die „Urteile“ dieser Öffentlichkeiten mit realen Kosten (z.B. internationalen Sanktionen oder innenpolitischer Machtverlust) verbunden sind, setzen sich paradoxerweise die Regeln verständigungsorientierten Handelns durch. 
Zugleich haben die kommunikativen Auseinandersetzungen um die Regeleinhaltung konkrete Folgen für das Verhalten der Beteiligten, entweder im Sinne der schrittweisen Verbesserung der Menschenrechtssituation oder als Machtverlust bzw. Regimewechsel im Falle fortwährenden Regelverstosses.

Damit zeigt auch das Beispiel der internationalen Menschenrechte, dass sich die oben beschriebenen Mechanismen der Regeldurchsetzung, die aus rationalistischer und aus konstruktivistischer Perspektive thematisiert werden, gegenseitig ergänzen und wechselseitig aufeinander beziehen. Insgesamt dominiert allerdings die vor allem von der konstruktivistischen Perspektive thematisierte Sozialisationsdynamik, was mit dem besonderen Charakter der Menschenrechte als konstitutiv für moderne Staatlichkeit zusammenhängt. Den Ausgangspunkt bilden internationale Normen mit hoher institutioneller Legitimität, die von transnational operierenden NormUnternehmern dazu benutzt werden, Regelverletzungen repressiver Regime vor einer internationalen Öffentlichkeit anzuprangern. Diese Norm-Unternehmer operieren zunächst durchaus strategisch, und die Reaktion der normverletzenden Regierungen folgt ebenfalls einer rationalistischen Handlungslogik. Die internationalen Normen sowie der Druck der Weltgesellschaft führt gleichzeitig zu einer Umverteilung ideeller und materieller Ressourcen zugunsten gesellschaftlicher Oppositionsgruppen gegen die repressiven Eliten. Nach der (verbalen) Normanerkennung durch die menschenrechtsverletzenden Regierungen und in der Phase taktischer Konzessionen setzen schliesslich die verschiedenen Internalisierungsmechanismen ein; argumentative Kommunikation wird immer wichtiger. Schließlich kommt es zur Normeinhaltung über die rechtliche Internalisierung und die Institutionalisierung standardisierter Verfahren des Menschenrechtsschutzes.

\section{Schlußbemerkungen und Forschungsdesiderate}

Die beiden Fallstudien sollten verdeutlichen, dass die von uns aus der Literatur abgeleiteten und auf zwei (meta-) theoretische Perspektiven zurückgeführten kausalen Mechanismen der Regeldurchsetzung in so unterschiedlichen Politikbereichen wie dem EU-Umweltrecht einerseits und der internationalen Menschenrechtspolitik andererseits wirksam werden. Die Ergebnisse sind in Abbildung 4 zusammengefasst, wobei hier allerdings noch einmal der illustrative Charakter der Fallstudien zu betonen ist. Es ging uns um einen Plausibilitäts-, nicht um einen Hypothesentest. 


\begin{tabular}{|c|c|c|}
\hline & $\begin{array}{l}\text { Europäische } \\
\text { Umweltpolitik }\end{array}$ & $\begin{array}{l}\text { Internationale } \\
\text { Menschenrechte }\end{array}$ \\
\hline \multicolumn{3}{|l|}{ 1) „Rationalistische“ Mechanismen } \\
\hline $\begin{array}{l}\text { a) Legalisierung: } \\
\text { - Detailliertheit der Normen } \\
\text { - materielle/ideelle Sanktionen }\end{array}$ & $\begin{array}{l}\text { Sehr wichtig } \\
\text { Bedingt wichtig }\end{array}$ & $\begin{array}{c}\text { Unwichtig } \\
\text { Bedingt wichtig }\end{array}$ \\
\hline b) Management-Hypothese & Wichtig & Unwichtig \\
\hline $\begin{array}{l}\text { c) Liberal-institutionenzentrierte } \\
\text { Hypothese }\end{array}$ & $\begin{array}{c}\text { Wichtig } \\
\text { wenn de facto Vetospieler } \\
\text { berücksichtigt werden }\end{array}$ & Falsifiziert \\
\hline $\begin{array}{l}\text { d) Liberal-akteurszentrierte } \\
\text { Hypothese }\end{array}$ & Sehr wichtig & Sehr wichtig \\
\hline \multicolumn{3}{|l|}{$\begin{array}{l}\text { 2) „Konstruktivistische“ } \\
\text { Mechanismen }\end{array}$} \\
\hline a) Verfahrenslegitimität & Wichtig & Unwichtig \\
\hline b) Institutionelle Legitimität & Falsifiziert & Wichtig \\
\hline c) Rechtliche Internalisierung & Bedingt wichtig & Sehr wichtig \\
\hline d) Soziale Internalisierung & Unwichtig & Sehr wichtig \\
\hline
\end{tabular}

Dabei ergibt sich, dass die Wirkungsmechanismen, die im Fall des europäischen Umweltrechts dominieren, im Fall der internationalen Menschenrechte eher unwichtig zu sein scheinen, und umgekehrt. Bei den Menschenrechten spielt danach der „konstruktivistische“ Kausalpfad eine herausragende Rolle, im europäischen Umweltrecht dagegen der „rationalistische“. Lediglich die liberal-akteurszentrierte These, wonach internationale Normen zu einer Umverteilung von materiellen und ideellen Ressourcen zugunsten gesellschaftlicher Reformkoalitionen führen, konnte in beiden Fällen als sehr wichtig bestätigt werden.

Dieses unterschiedliche Bild dürfte allerdings im wesentlichen mit der Fallauswahl zusammenhängen. Bei der Durchsetzung grundlegender Menschenrechten in autoritären Regimen geht es um konstitutive Normen, um die grundlegende Veränderung von Staatlichkeit und staatlicher Identität sowie die allmähliche Verwirklichung von Rechtsstaatlichkeit als einer Vorbedingung von Regeleinhaltung (sei es national oder international). Insofern ist es kein Wunder, dass hier rechtlichen und sozialen Internalisierungsprozessen eine herausragende Bedeutung zukommt, weil nur sie - im Unterschied zu den „rationalistischen“ Mechanismen - in der Lage sind, die erforderlichen Präferenz- und Identitätsveränderungen zu bewirken. Das europäische Gemeinschaftsrecht hingegen trifft auf stabile liberale Demokratien, in denen Rechtsstaatlichkeit und die Legitimität rechtsstaatlicher Verfahren nicht (mehr) in Frage stehen bzw. zu den Hintergrundbedingungen der Regeldurchsetzung gehören. Die rechtliche Internalisierung des Gemeinschafts- 
rechts ist zudem weit fortgeschritten. Im EU-Umweltrecht geht es daher vor allem um die Einhaltung regulativer Normen, obwohl auch hier der ,misfit“ mit nationalem Recht zum Teil konstitutiv begründet ist, etwa aufgrund unterschiedlicher Regulierungsstile oder Verwaltungstraditionen (vgl. dazu auch Knill und Lenschow 2001).

Vor diesem Hintergrund ist es nicht allzu erstaunlich, dass vor allem die von der rationalistischen Perspektive thematisierten Mechanismen der Regeldurchsetzung im EU-Umweltrecht zu dominieren scheinen. Wenn die Legitimität der entsprechenden Normen nicht (mehr) umstritten ist, dann muss die Varianz bei der Normeinhaltung in erster Linie mit den unterschiedlichen Nutzenkalkülen der Akteure erklärt werden, so dass den von der rationalistischen Perspektive thematisierten Kausalpfaden Priorität zukommt. Im Menschenrechtsbereich dagegen geht es bei den hier untersuchten Fällen um die schrittweise Durchsetzung einer neuen „Logik der Angemessenheit“. Dementsprechend gewinnen Sozialisationsdynamiken eine größere Erklärungskraft.

Eine weitere Differenz der beiden Politikbereiche kommt hinzu. Im europäischen Umweltrecht fallen Regeladressaten und -zielgruppen häufig auseinander, insofern die Verhaltensänderungen von privaten Akteuren zu erbringen sind. Dementsprechend kommt es hier häufig zu ungewollten Regelverstößen, die wiederum eher von der Management-Perspektive der complianceForschung thematisiert werden. Auch das völlig unterschiedliche Abschneiden der liberalinstitutionenzentrierten Hypothese, wonach die Regelbefolgung mit größerer Autonomie der Regierungen gegenüber ihren gesellschaftlichen Umfeldern zunimmt, lässt sich so erklären. Im Menschenrechtsbereich hingegen geht es vor allem um gewollte Normenverletzungen, weil Regeladressaten und -zielgruppen die gleichen Akteure, nämlich nationale Regierungen und ihre Organe sind, zumindest in den hier untersuchten Fällen. Außerdem zielen Menschenrechte auf die Einhegung und Kontrolle staatlicher Herrschaft ab, nicht auf die Vergrößerung staatlicher Autonomie.

Internationale Menschenrechte und europäisches Umweltrecht stellen daher höchst unterschiedliche Fälle für die Erforschung der Normeinhaltung dar. Umso erstaunlicher sind zwei Ergebnisse, die für beide Politikbereiche gelten. Erstens spielen in beiden Politikbereichen rein materielle Sanktionen nur eine geringe Rolle bei der Regeldurchsetzung, und auch nur dann, wenn sie in andere Mechanismen eingebettet sind. Ökonomische und militärische Sanktionen gegen Schurkenstaaten sind notorisch ungeeignet, den Menschenrechten zum Durchbruch zu verhelfen. Und im EU-Umweltrecht sind Drohungen mit Strafzahlungen von untergeordneter Bedeutung für die Regeldurchsetzung. Soweit die Regelverletzungen in beiden Fällen überhaupt einen Preis haben, handelt es sich in erster Linie um immaterielle, nämlich politische, rechtliche und symbolische Kosten. Diese Kosten ergeben sich für die Akteure aber erst dann, wenn die Legitimität der Regeln und der Verfahren ihrer Durchsetzung selbst nicht in Frage stehen. Im Falle der EU lässt sich anders nicht erklären, warum die meisten Regierungen spätestens dann die Regeln einhalten, wenn ihnen ein Verfahren vor dem EuGH droht, der erst seit 1997 die Möglichkeit hat, GeldbuBen für die Nichteinhaltung seiner Urteile zu verhängen. Im Bereich der Menschenrechte sind es vor allem die symbolischen Kosten internationaler Isolation, die in vielen Fällen zu taktischen Konzessionen durch autoritäre Regime führen. 
Zweitens zeigt sich in beiden Fällen die überragende Bedeutung gesellschaftlicher Reformkoalitionen bei der Regeleinhaltung. Dabei läßt sich oft empirisch nicht unterscheiden, ob die Durchsetzungkraft dieser Reformkoalitionen über den rationalistischen Kausalpfad der Ressourcenumverteilung verläuft oder über den konstruktivistischen Mechanismus der strategischen Konstruktionen und argumentativen Überzeugung. Dies genauer zu untersuchen, erscheint uns als ein wichtiges Desiderat für die zukünftige Forschung. Ein weiteres Desiderat besteht in der präziseren Beschreibung der argumentativen Kommunikationsprozesse, die für die Normdurchsetzung von herausragender Bedeutung zu sein scheinen. 


\section{Literatur}

Abbott, Kenneth W., Robert O. Keohane, Andrew Moravcsik, Anne-Marie Slaughter, und Duncan Snidal 2000. The Concept of Legalization. International Organization 54: Nr. 3, 401419.

Abbott, Kenneth W., und Duncan Snidal 2000. Hard and Soft Law in International Governance. International Organization 54: Nr. 3, 421-456.

Alter, Karen J. 2000. The European Union's Legal System and Domestic Policy: Spillover or Backlash? International Organization 54: Nr. 3, 489-518.

Börzel, Tanja A. 2000. Why There Is No Southern Problem. On Environmental Leaders and Laggards in the European Union. Journal of European Public Policy 7: Nr. 1, 141-162.

Börzel, Tanja A. 2001. Non-Compliance in the European Union. Pathology or Statistical Artifact?. Journal of European Public Policy 8: Nr. 5, 803-824.

Börzel, Tanja A. i.E. On Environmental Leaders and Laggards in the European Union. Why There is (Not) a Southern Problem, London.

Breitmeier, Helmut, und Klaus Dieter Wolf 1995. Analysing Regime Consequences. Conceptual Outlines and Environmental Explorations. In Regime Theory and International Relations, hrsg. von Volker Rittberger und Peter Mayer, Oxford.

Bull, Hedley 1977. The Anarchical Society. A Study of Order in World Politics, New York.

Burley, Anne-Marie, und Walter Mattli 1993. Europe before the Court: A Political Theory of Legal Integration. International Organization 47: Nr. 1, 41-77.

Chayes, Abram, Antonia Handler Chayes, und Ronald B. Mitchell 1998. Managing Compliance: A Comparative Perspective. In Engaging Countries: Strengthening Compliance with International Environmental Accords, hrsg. von Edith Brown Weiss und Harold K. Jacobsen, Cambridge, Mass., 39-62.

Chayes, Abram, und Antonia Chayes Handler 1993. On Compliance. International Organization 47: Nr. 2, 175-205.

Chayes, Abram, und Antonia Chayes Handler 1995. The New Sovereignty. Compliance with International Regulatory Agreements, Cambridge, MA.

Checkel, Jeffrey T. 1997. International Norms and Domestic Politics: Bridging the RationalistConstructivist Divide. European Journal of International Relations 3: Nr. 4, 473-495.

Checkel, Jeffrey T. 1999. International Institutions and Socialization. Working Paper. 5. Oslo: ARENA, University of Oslo, February. 
Conant, Lisa J. 2001. Europeanization and the Courts: Variable Patterns of Adaptation among National Judiciaries. In Transforming Europe. Europeanization and Domestic Change. hrsg. von Maria Green Cowles, James A. Caporaso und Thomas Risse. Ithaca, NY, 97-115.

Commission of the European Communities 1991. Monitoring of the Application by Member States of Environment Directives. Annex C to the Eighth Annual Report to the European Parliament on the Application of Community Law. Official Journal of the European Communities C 338: Nr. 31.12.1991.

Cortell, Andrew P., und James W. Jr. Davis 2000. Understanding the Domestic Impact of International Norms: A Research Agenda. International Studies Review 2: Nr. 1, 65-87.

Cowles, Maria Green, James Caporaso, und Thomas Risse, (Hrsg.) 2001. Transforming Europe: Europeanization and Domestic Change, Ithaca NY.

Downs, George W., David M. Rocke, und Peter N. Barsoom 1996. Is the Good News About Compliance Good News About Cooperation? International Organization 50: Nr. 3, 379406.

Drezner, Daniel W. 1999. The Sanctions Paradox: Economic Statecraft and International Relations, Cambridge.

Drezner, Daniel W. 2000. Bargaining, Enforcement, and Multilateral Sanctions: When Is Cooperation Counterproductive? International Organization 54: Nr. 1, 73-102.

Dworkin, Ronald 1986. Law’s Empire, Cambridge, MA.

Elliott, Kimberly Ann 1998. The Sanctions Glass. Half Full or Completely Empty? International Security 23: Nr. 1, 50-65.

Evans, Peter B., Harold K. Jacobson, und Robert D. Putnam, (Hrsg.) 1993. Double-Edged Diplomacy: International Bargaining and Domestic Politics, Berkeley CA.

Finnemore, Martha 1996. National Interests in International Society, Ithaca.

Finnemore, Martha, und Kathryn Sikkink 1998. International Norm Dynamics and Political Change. International Organization 52: Nr. 4, 887-917.

Forschungsgruppe Menschenrechte 1998. Internationale Menschenrechtsnormen, transnationale Netzwerke und politischer Wandel in den Ländern des Südens. Zeitschrift für Internationale Beziehungen 5: Nr. 1, 5-41.

Franck, Thomas M. 1990. The Power of Legitimacy Among Nations, Oxford.

Franck, Thomas M. 1995. Fairness in International Law and Institutions, Oxford. 
Gehring, Thomas 1994. Der Beitrag von Institutionen zur Förderung der internationalen Zusammenarbeit. Zeitschrift für Internationale Beziehungen 1: Nr. 2, 211-242.

Goldstein, Judith 1996. International law and domestic institutions: reconciling North American "unfair" trade laws. International Organization 50: Nr. 4, 541-564.

Haas, Peter M. 1998. Compliance With EU Directives: Insights from International Relations and Comparative Politics. Journal of European Public Policy 5: Nr. 1, 17-37.

Habermas, Jürgen 1981. Theorie des kommunikativen Handelns, Frankfurt/M.

Habermas, Jürgen 1992. Faktizität und Geltung. Beiträge zur Diskurstheorie des Rechts und des demokratischen Rechtsstaats, Frankfurt/M.

Hallo, Ralph 1996. Directive 90/313/EEC on the Freedom of Access to Information on the Environment: Its Implementation and Implications. In Access to Environmental Information in Europe. The Implementation and Implications of Directive 90/313/EEC, hrsg. von Ralph Hallo, London, The Hague, Boston, 1-26.

Haverland, Markus 1999. National Autonomy, European Integration, and the Politics of Packaging Waste, Amsterdam.

Hazelzet, Hadewych 2001. Carrots or Sticks? EU and US Sanctions in Reaction to Human Rights Violations. PhD. dissertation, European University Institute.

Héritier, Adrienne 1996. The accommodation of diversity in European policy-making. Journal of European Public Policy 3: Nr. 2, 149-76.

Héritier, Adrienne, Susanne Mingers, Christoph Knill, und Martina Becka 1994. Die Veränderung von Staatlichkeit in Europa. Ein regulativer Wettbewerb: Deutschland, Großbritannien und Frankreich in der Europäischen Union, Opladen.

Hufbauer, Gary Clyde, Jeffrey J. Schott, und Kimberly Ann Elliott 1990. Economic Sanctions Reconsidered: History and Current Policy, Washington DC.

Hurd, Ian 1999. Legitimacy and Authority in International Politics. International Organization 53: Nr. 2, 379-408.

Hurrell, Andrew 1993. International Society and the Study of Regimes. A Reflective Approach. In Regime Theory and International Relations, hrsg. von Volker Rittberger, Oxford, 49-72.

Jacobson, Harold K., und Edith Brown Weiss 1995. Improving Compliance with International Environmental Accords. Global Governance 1: Nr. 2, 119-149.

Jänicke, Martin, und Helmut Weidner, (Hrsg.) 1997. National Environmental Policies. A Comparative Study of Capacity-Building, Berlin et al. 
Jetschke, Anja 1999. Linking the unlinkable? International norms and nationalism in Indonesia and the Philippines. In The Power of Human Rights: International Norms and Domestic Change, hrsg. von Thomas Risse, Stephen C. Ropp und Kathryn Sikkink, Cambridge, 134171.

Jetschke, Anja 2000. International Norms, Transnational Human Rights Networks, and Domestic Political Change in Indonesia and the Philippines. PhD. dissertation, Florence: European University Institute.

Joerges, Christian, und Jürgen Neyer 1997. Transforming strategic interaction into deliberative problem-solving: European comitology in the foodstuffs sector. Journal of European Public Policy 4, 609-625.

Katzenstein, Peter J. 1984. Corporatism and Change, Ithaca NY.

Katzenstein, Peter J., (Hrsg.) 1978. Between Power and Plenty: Foreign Economic Policies of Advanced Industrial States, Madison.

Keck, Margaret, und Kathryn Sikkink 1998. Activists Beyond Borders: Advocacy Networks in International Politics, Ithaca NY.

Keck, Otto 1995. Rationales kommunikatives Handeln in den internationalen Beziehungen. Ist eine Verbindung von Rational-Choice-Theorie und Habermas' Theorie des kommunikativen Handelns möglich? Zeitschrift für Internationale Beziehungen 2: Nr. 1, 5-48.

Keith, Linda Camp 1999. The United Nations International Covenant on Civil and Political Rights: Does It Make a Difference in Human Rights Behavior? Journal of Peace Research 36: Nr. 1, 95-118.

Keohane, Robert O. 1984. After Hegemony. Cooperation and Discord in the World Political Economy, Princeton NJ.

Knill, Christoph, und Andrea Lenschow 2000. New Concepts - Old Problems? The Institutional Constraints for the Effective Implementation of EU Environmental Policy. In Implementing EU Environmental Policy: New Approaches to an Old Problem, hrsg. von Christoph Knill und Andrea Lenschow, Manchester.

Knill, Christoph, und Andrea Lenschow 2001. Adjusting to EU Environmental Policy: Change and Persistence of Domestic Administrations. In Transforming Europe. Europeanization and Domestic Change, hrsg. von Maria Green Cowles, James A. Caporaso und Thomas Risse, Ithaca, NY, 116-136.

Koh, Harold Hongju 1997. Why Do Nations Obey International Law? The Yale Law Journal 106, 2599-2659. 
Kohler-Koch, Beate 1993. Die Welt regieren ohne Weltregierung. In Regieren im 21. Jahrhundert - Zwischen Globalisierung und Regionalisierung. Festgabe für Hans-Hermann Hartwich zum 65. Geburtstag, hrsg. von C. Böhret und G. Wewer, Opladen, 109-141.

Kohler-Koch, Beate 1994. Changing patterns of interest intermediation in the European Union. Government and Opposition 29: Nr. 2.

Kohler-Koch, Beate 1996. The Strength of Weakness. The Transformation of Governance in the EU. In The Future of the Nation State. Essays on Cultural Pluralism and Political Integration, hrsg. von Sverker Gustavsson und Leif Lewin, Stockholm, 169-210.

Kohler-Koch, Beate 1998a. Europäisierung der Regionen: Institutioneller Wandel als sozialer Prozeß. In Interaktive Politik in Europa. Regionen im Netzwerk der Integration, hrsg. von Beate et al. Kohler-Koch, Opladen, 13-31.

Kohler-Koch, Beate, (Hrsg.) 1998b. Interaktive Politik in Europa. Regionen im Netzwerk der Integration, Opladen.

Kohler-Koch, Beate, (Hrsg.) 1998c. Regieren in entgrenzten Räumen, PVS-Sonderheft. Vol. 29, Opladen.

Kohler-Koch, Beate, und Rainer Eising, (Hrsg.) 1999. The Transformation of Governance in the European Union, London.

Kratochwil, Friedrich 1989. Rules, Norms, and Decisions, Cambridge.

Legro, Jeff W. 1996. Culture and Preferences in the International Cooperation Two-Step. Americal Political Science Review 90: Nr. 1, 118-137.

Levy, Marc A., Robert O. Keohane, und Peter M. Haas 1993. Improving the Effectiveness of International Environmental Institutions. In Institutions for the Earth: Sources of Effective International Environmental Protection, hrsg. von Peter M. Haas, Robert O. Keohane und Marc A. Levy, Cambridge, Mass., 397-426.

Liese, Andrea 2001. Staaten am Pranger. Zur Wirkung internationaler Regime auf die innerstaatliche Menschenrechtspolitik. PhD. dissertation, Universität Bremen.

Lutz, Ellen L., und Kathryn Sikkink 2000. International Human Rights Law and Practice in Latin America. International Organization 54: Nr. 3, 633-659.

Marauhn, Thilo 1996. Towards a Procedural Law of Compliance Control in International Environmental Relations. Zeitschrift für Ausländisches Öffentliches Recht und Völkerrecht 56: Nr. 2, 686-731.

March, James G., und Johan P. Olsen 1989. Rediscovering Institutions, New York. 
March, James G., und Johan P. Olsen 1998. The Institutional Dynamics of International Political Orders. International Organization 52: Nr. 4, 943-969.

Meyer, John, und Brian Rowen 1991. Institutionalized Organizations: Formal Structures in Myth and Ceremony. In The New Institutionalism in Organizational Analysis, hrsg. von Paul J. Dimaggio und Walter Powell, Chicago.

Meyer, John W., John Boli, und George Thomas 1987. Ontology and Rationalization in the Western Cultural Account. In Institutional Structure: Constituting State, Society, and the Individual, hrsg. von George Thomas und et al., Newbury Park CA, 12-37.

Mills, Geofrey, und Hugh Rockoff 1987. Compliance with Prioce Controls in the United States and the United Kingdom during World War II. Journal of Economic History 47: Nr. 1, 197-213.

Milner, Helen V. 1988. Resisting Protectionism: Global Industries and the Politics of International Trade, Princeton.

Mitchell, Ronald B. 1994a. Intentional Oil Pollution at Sea. Environmental Policy and Treaty Compliance, Cambridge, MA.

Mitchell, Ronald B. 1994b. Regime Design Matters: Intentional Oil Pollution and Treaty Compliance. International Organization 48: Nr. 3, 425-458.

Mitchell, Ronald B. 1996. Compliance Theory: An Overview. In Improving Compliance with International Environmental Law, hrsg. von James Cameron, Jacob Werksman und Peter Roderick, London, 3-28.

Moravcsik, Andrew 1997. Warum die Europäische Union die Exekutive stärkt: Innenpolitik und internationale Kooperation. In Projekt Europa im Übergang? Staat und Demokratie in der Europäischen Union, hrsg. von Klaus Dieter Wolf, Baden-Baden.

Moravcsik, Andrew 2000. The Origins of Human Rights Regimes: Democratic Delegation in Postwar Europe, in: International Organization 54: Nr. 2, 217-252.

Müller, Harald 1994. Internationale Beziehungen als kommunikatives Handeln. Zur Kritik der utilitaristischen Handlungstheorien. Zeitschrift für Internationale Beziehungen 1: Nr. 1, 1544.

Odell, John S. i.E. Bounded Rationality and the World Political Economy. In Organizing the World Economy. Essays in Honor of Benjamin J. Cohen, hrsg. von David Andrews, Randall Henning und Louis Pauly.

O'Donnell, Guillermo, und Philippe C. Schmitter 1986. Transitions from Authoritarian Rule. Tentative Conclusions about Uncertain Democracies, Baltimore. 
Onuf, Nicholas 1989. World of Our Making: Rules and Rule in Social Theory and International Relations, Columbus SC.

Ponce-Nava, Diana 1995. Capacity Building in Environmental Law and Sustainable Development. In Sustainable Development and International Law, hrsg. von Winfried Lang, London, 131-136.

Putnam, Robert 1988. Diplomacy and Domestic Politics. The Logic of Two-Level Games. International Organization 42: Nr. 2, 427-460.

Raustiala, Kal, und Anne-Marie Slaughter 2002. Considering Compliance. In Handbook of International Relations, hrsg. von Walter Carlsnaes, Beth Simmons und Thomas Risse, London et al.

Raustiala, Kal 1997. Domestic Institutions and International Regulatory Cooperation. Comparative Responses to the Convention of Biological Diversity. World Politics 49: Nr. 4, 482509.

Risse, Thomas 1999. International Norms and Domestic Change: Arguing and Communicative Behavior in the Human Rights Area. Politics \& Society 27: Nr. 4, 526-556.

Risse, Thomas 2000. 'Let's Argue!' Communicative Action in International Relations. International Organization 54: Nr. 1, 1-39.

Risse, Thomas i.E. Rationalism, Constructivism, and the Study of International Institutions: An Emerging Synthesis? In Political Science as Discipline? Reconsidering Power, Choice, and the State at Century's End, hrsg. von Ira Katznelson und Helen V. Milner.

Risse, Thomas, Anja Jetschke, und Hans-Peter Schmitz i.E. Die Macht der Menschenrechte. Internationale Normen, kommunikative Prozesse und politischer Wandel in den Ländern des Südens, Baden-Baden

Risse, Thomas, Stephen C. Ropp, und Kathryn Sikkink, (Hrsg.) 1999. The Power of Human Rights: International Norms and Domestic Change, Cambridge.

Rogowski, Ronald 1989. Commerce and Coalitions: How Trade Affects Domestic Political Alignments, Princeton, NJ.

Sandholtz, Wayne 1996. Membership Matters: Limits of the Functional Approach to European Institutions. Journal of Common Market Studies 34: Nr. 3, 403-429.

Scharpf, Fritz W. 1997. Games Real Actors Play. Actor-Centered Institutionalism in Policy Research, Boulder CO.

Schimmelfennig, Frank 1997. Rhetorisches Handeln in der internationalen Politik. Zeitschrift für internationale Beziehungen 4: Nr. 2, 219-254. 
Schimmelfennig, Frank 2001. The Community Trap: Liberal Norms, Rhetorical Action, and the Eastern Enlargement of the European Union. International Organization 55: Nr. 1, 81-122.

Schmidt, Vivien A. 1996. From State to Market? The Transformation of French Business and Government, Cambridge.

Simmons, Beth A., und Lisa Martin 2002. International Organisations, Institutions, and Governance. In Handbook of International Relations, hrsg. von Walter Carlsnaes, Thomas Risse und Beth Simmons, London et al.

Simon, Herbert A. 1982. Models of Bounded Rationality. Volume 1. Economic Analysis and Public Policy. Volume 2. Behavioral Economics and Business Organization, Cambridge MA.

Simon, Herbert A. 1997. Models of Bounded Rationality. Volume 3. Empirically Grounded Economic Reason, Cambridge MA.

Slaughter, Anne-Marie 1995. International Law in a World of Liberal States. European Journal of International Law 6, 504-538.

Snyder, Francis 1993. The Effectiveness of European Community Law. Institutions, Processes, Tools and Techniques. Modern Law Review: Nr. 56, 19-54.

Stone Sweet, Alec, und Thomas L. Brunell 1998. Constructing a Supranational Constitution: Dispute Resolution and Governance in the European Community. American Political Science Review 92: Nr. 1, 63-81.

Tsebelis, George 1995. Decision Making in Political Systems. Veto Players in Presidentialism, Parliamentarism, Multicameralism and Multipartism. British Journal of Political Science 25: Nr. 3, 289-325.

Ulbert, Cornelia 1997. Die Konstruktion von Umwelt. Der Einfluss von Ideen, Institutionen und Kultur auf (inter-)nationale Klimapolitik in den USA und der Bundesrepublik, BadenBaden.

Underdal, Arild 1998. Explaining Compliance and Defection: Three Models. European Journal of International Relations 4: Nr. 1, 5-30.

Victor, David G., Kal Raustiala, und Eugene B. Skolnikoff, (Hrsg.) 1998. The Implementation and Effectiveness of International Environmental Commitments, Cambridge, MA.

Wendt, Alexander 1999. Social Theory of International Politics, Cambridge.

Young, Oran R. 1979. Compliance and Public Authority: A Theory with International Applications, Baltimore, MD. 
Zangl, Bernhard, und Michael Zürn 1996. Argumentatives Handeln bei internationalen Verhandlungen. Moderate Anmerkungen zur post-realistischen Debatte. Zeitschrift für Internationale Beziehungen 3: Nr. 2, 341-366.

Zürn, Michael 1997. "Positives Regieren” jenseits des Nationalstaates. Zeitschrift für Internationale Beziehungen 4: Nr. 1, 41-68.

Zürn, Michael, und Christian Joerges 1999. The Study of Compliance - A Framework. Manuscript, Bremen-Florenz. 